\title{
Nematóides do Brasil. Nematóides de peixes Atualização: 1985-1998
}

\author{
Joaquim Júlio Vicente ${ }^{1}$ \\ Roberto Magalhães Pinto ${ }^{1}$
}

\begin{abstract}
Brazilian Nematodes. Nematodes of fishes - updating: 1985-1998. Fifty-three nematode species, related to 9 superfamilies, 13 families and 26 genera, recovered from Brazilian fishes, represented by 75 host species are presented. Thirtyeight nematode species, out of the 53 reported, are also illustrated and included in measurement tables, one is listed and figured, and two only listed. Data on family, genus and subgenus level, are provided.

KEY WORDS. Nematodes, fishes, Brazil
\end{abstract}

\section{CONTEÚDO}

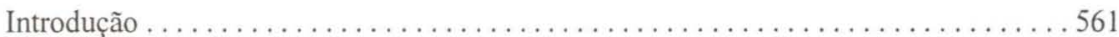

Posição sistemática das espécies catalogadas . . . . . . . . . . . . . . . . . . . 562

Caracteres diagnósticos de famílias, gêneros e subgêneros não incluídos em Vicente et al.

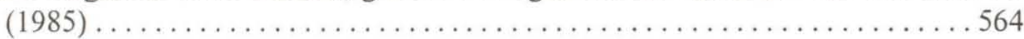

Famílias, gêneros e/ou espécies não catalogadas em VICENTE et al. (1985) ........... 565 Espécies catalogadas (VICENTE et al. 1985) em outros hospedeiros ............. 578

Lista alfabética dos hospedeiros e seus respectivos nematóides . . . . . . . . . . . . 580

Referências bibliográficas ................................... 585

Tabelas ....................................................... 589

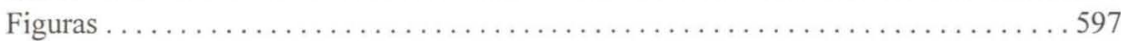

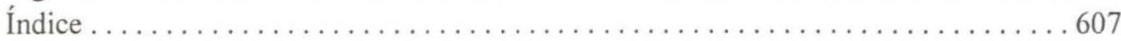

Tendo em vista as várias espécies de nematóides parasitas de peixes do Brasil que vem sendo propostas desde que VICENTE et al. (1985) catalogaram este grupo de helmintos parasitos de peixes brasileiros, julgamos necessário e oportuno, atualizar os dados a partir daquela data.

A organização sistemática dos nematóides incluídos no presente trabalho é a proposta por Hartwich (1974), Chabaud (1975a,b), PetTer \& Quentin (1976), Chabaud (1978) e ANDERSON \& BAIN (1982).

Com relação às espécies de peixes hospedeiros, elas são referidas conforme aparecem nos trabalhos originais. Além disso, são apresentados caracteres diagnósticos para famílias, gêneros e subgêneros não relacionados por VICENTE et al. (1985).

1) Laboratório de Helmintos Parasitos de Vertebrados, Departamento de Helmintologia, Instituto Oswaldo Cruz. Caixa Postal 926, 21045-900 Rio de Janeiro, Rio de Janeiro, Brasil. Bolsista do CNPq. 


\section{POSIÇÃO SISTEMÁTICA DAS ESPÉCIES CATALOGADAS}

Trichinelloidea

Trichuridae

Trichuridae sp. de MORAVEC et al. (1992)

Capillaria Zeder, 1800

Capillaria sp. de KoHN et al. (1988)

Dioctophymatoidea

Dioctophymatidae

Eustrongylides Jägerskiöld, 1909

Eustrongylides ignotus Jägerskiöld, 1909

Oxyuroidea

Pharyngodonidae

Brasilnema Moravec, Kohn \& Fernandes, 1992

Brasilnema pimelodellae Moravec, Kohn \& Fernandes, 1992

Travnema Pereira, 1938

Travnema travnema Pereira, 1938 (MORAVEC et al. 1992b, 1994b)

Cosmoxynema Travassos, 1948

Cosmoxynemoides aguirrei Travassos, 1949 (MORAVEC et al. 1992b)

Parasynodontisia Moravec, Kohn \& Fernandes, 1992

Parasynodontisia petterae Moravec, Kohn \& Fernandes, 1992

Ichthyouris Inglis, 1962

Ichthyouris laterifilamenta Moravec, Kohn \& Fernandes, 1992

Cosmocercoidea

Atractidae

Rondonia Travassos, 1919

Rondonia rondoni Travassos, 1919 (MORAVEC et al. 1992b)

Kathlaniidae

Spectatus Travassos, 1913

Spectatus sp. de MORAVEC et al. (1997)

Seuratoidea

Cucullanidae

Cucullanus Müller, 1777

Cucullanus cassinensis Pereira \& Costa, 1996

Cucullanus debacoi Sarmento, Fortes \& Hoffmann, 1995

Cucullanus grandistomis (Ferraz \& Thatcher, 1988) Moravec, Kohn \& Fernandes, 1993 (= Bacudactinis grandistomis)

Cucullanus pinnai Travassos, Artigas \& Pereira, 1928

Cucullanus patoi Fortes, Hoffmann \& Sarmento, 1992

Cucullanus fabregasi Fortes, Hoffmann \& Sarmento, 1993

Cucullanus riograndensis Fortes, Hoffmann \& Sarmento, 1992

Cucullanus pseudoplatystomae Moravec, Kohn \& Fernandes, 1993

Cucullanus brevispiculus Moravec, Kohn \& Fernandes, 1993

Cucullanus pimelodellae Moravec, Kohn \& Fernandes, 1993

Cucullanus pinnai pinnai Travassos, Artigas \& Pereira, 1928

Cucullanus pinnai pterodorasi Moravec, Kohn \& Fernandes, 1997

Revta bras. Zool. 16 (3): 561 - 610, 1999 
Cucullamus pulcherrimus Barreto, 1918

Cucullanus rhamphichthydis Moravec, Kohn \& Fernandes, 1997

Cucullanus zungaro Vaz \& Pereira, 1934

Cucullamis sp. de MORAVEC et al. (1993)

Dichelyne Jägerskiöld, 1902

Dichelyne (Cucullanellus) amaruincai (Freitas, Vicente \& Ibañez,1969) Petter, 1974

Dichelyne (Cucullanellus) elongatus (Törnquist, 1931) Petter, 1974

Dichelyne leporini Petter, 1989

Dichelyne (Cucullanellus) micropogonii Pereira \& Costa, 1996

Dichelyne pimelodi Moravec, Kohn \& Fernandes, 1997

Dichelyne (Cucullanellus) sp. de PINTO et al. (1992)

Quimperiidae

Neoparaseuratum Moravec, Kohn \& Fernandes, 1992

Neoparaseuratum travassosi Moravec, Kohn \& Fernandes, 1992

Seuratidae

Seuratoidea sp. de MORAVEC et al. (1997)

Ascaridoidea

Anisakidae

Contracaecum Railliet \& Henry, 1912

Contracaecum sp. de Korn et al. (1988)

Goezia Zeder, 1800

Goezia brasiliensis Moravec, Kohn \& Fernandes, 1994

Goezia brevicaeca Moravec, Kohn \& Fernandes, 1994

Goezia sp. de MoRAVEC et al. (1994)

Hysterothylacium Ward \& Magath, 1916

Hysterothylacium sp. de RÉGO et al. (1985)

Hysterothylacium sp. de MORAVEC et al. (1993)

Raphidascaris Yamaguti, 1941

Raphidascaris (Sprentascaris) hypostomi (Petter \& Cassone, 1984) Moravec, Kohn \& Fernandes, 1990

Raphidascaris (S.) mahnerti (Petter \& Cassone, 1984) Moravec, Kohn \& Fernandes, 1990

Raphidascaroides Yamaguti, 1941

Raphidascaroides brasiliensis Moravec \& Thatcher, 1997

Terranova Leiper \& Atkinson, 1914

Terranova sp. de RÉGo et al. (1985)

Ascarididae

Amplicaecum Baylis, 1920

Amplicaecum sp. de KOHN \& FERNANDES (1987)

Camallanoidea

Camallanidae

Camallamus Railliet \& Henry, 1915

Camallanus acaudatus Ferraz \& Thatcher, 1990

Camallanus tridentatus (Drasche, 1884) Railliet \& Henry, 1915

Paracamallanus Yorke \& Maplestone, 1926

Paracamallanus amazonensis Ferraz \& Thatcher, 1990 
Paracamallanus sp. de Thatcher (1991)

Procamallanus Baylis, 1923

Procamallanus (Procamallanus) annipetterae (Kohn \& Fernandes, 1988) Kohn \& Fernandes, 1988

Procamallanus (P.) freitasi (Moreira, Oliveira \& Costa, 1991) comb.n.

Procamallanus hilarii Vaz \& Pereira, 1934 (KoHn \& FERNANDEs 1987; MoraVEC et al. 1993)

Procamallanus (Spiricamallanus) inopinatus Travassos, Artigas \& Pereira, 1928 (KOHN \& FERNANDES 1987; MORAVEC et al. 1993)

Procamallanus (S.) iheringi Travassos, Artigas \& Pereira, 1928 (KoHn \& FERnANDES 1987; MORA VEC et al. 1993)

Procamallanus (P.) peraccuratus Pinto, Fabio, Noronha \& Rolas, 1976 (MorAVEC et al. 1993)

Procamallamus (S.) pimelodus Pinto, Fabio, Noronha \& Rolas, 1974 (= Procamallanus (S.) intermedius Pinto, Fabio, Noronha \& Rolas, 1974) (MORAvec et al. 1993)

Procamallanus (S.) saofranciscencis (Moreira, Oliveira \& Costa, 1994) comb.n.

Procamallanus sp. de KoHn \& FERNANDES (1987)

Oncophora Diesing, 1851

Oncophora melanocephala (Rudolphi, 1819) Baudin-Laurecin, 1971

Acuarioidea

Acuariidae

Acuariinae sp. de Moravec, Kohn \& Fernandes (1993)

Dracunculoidea

Guyanemidae

Guyanema Petter, 1974

Guyanema raphidioni Moravec, Kohn \& Fernandes, 1993

Guyanema sp. de MoRAvEC et al. (1993)

Travassosnema Costa, Pereira \& Oliveira, 1991

Travassosnema travassosi Costa, Pereira \& Oliveira, 1991

Travassosnema travassosi paranaensis Moravec, Kohn \& Fernandes, 1993

\section{CARACTERES DIAGNÓSTICOS DE FAMÍLIAS, GÊNEROS E SUBGÊNEROS NÃO INCLUIDOS EM VICENTE ET AL. (1985)}

\section{Oxyuroidea}

Machos com cauda truncada, um espículo e dotados de formações esclerotizadas e apêndice caudal delgado e longo..................... Pharyngodonidae (1-3)

1. Asas laterais presentes em ambos os sexos. Asas caudais ausentes. Cápsula bucal larga. Cauda com apêndice terminal muito longo em ambos os sexos. Ovos com longos

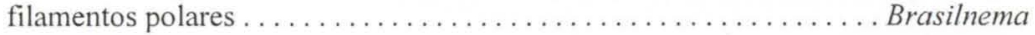

2. Asas laterais, caudais e cápsula bucal ausentes. Cauda cônica em ambos os sexos. Ovos

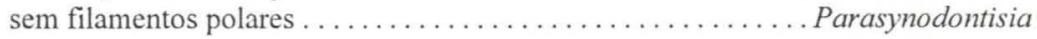

3. Parte anterior do esôfago extremamente modificada nas fêmeas. Asas caudais presentes,

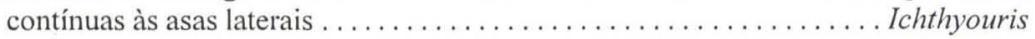

\section{Ascaridoidea - Anisakidae}

Boca com interlábios e fileiras de dentículos nos lábios . . . . . . . Raphidascaroides 


\section{Seuratoidea}

Sem cápsula bucal. Ventrículo e divertículo intestinal ausentes. Machos usualmente com ventosa pré-cloacal, sem anel esclerotizado ............ Quimperiidae (1)

1. Boca rodeada por seis cristas que se originam da margem bucal. Região cefálica do corpo rodeada por numerosas bandas longitudinais de cutícula inflada . Neoparaseuratum

\section{Cucullanidae}

Boca perpendicular ao eixo do corpo ou ligeiramente inclinada, sustentada por estrutura peribucal esclerotizada. Ceco intestinal presente. Ventosa pré-cloacal presente ou

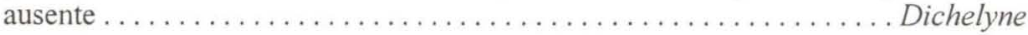

1.Ventosa pré-cloacal presente . . . . . . . . . . . . . . . . (Cucullanellus)

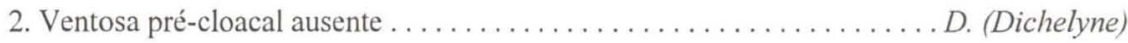

\section{Camallanoidea - Camallanidae}

1. Cápsula bucal formada por duas valvas laterais, divididas em dois níveis, com uma larga cavidade atrás das valvas . . . . . . . . . . . . . . . Paracamallanus

2. Valvas sustentadas por bandas longitudinais que se modificam posteriormente em fileiras de espinhos ou cerdas. Fêmeas maduras extremamente alargadas posteriormente . .

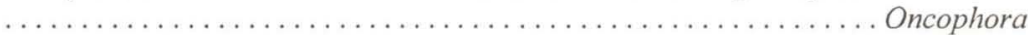

Observação. Em ambos os gêneros existe um par de processos esclerotizados em forma de tridente na junção das valvas.

\section{Dracunculoidea}

Extremidade apical com 4 papilas cefálicas e 8 labiais, sendo 4 internas e 4 externas. Tanto as papilas cefálicas quanto as labiais são deslocadas lateralmente. Ausência de

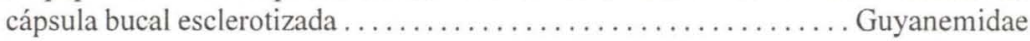

1. Com as mesmas características da família . . . . . . . . . . . . . . Guyanema

2. Boca oval, sem lábios. Esôfago glandular com apêndice posterior ........ Travassosnema

\section{FAMÍLIAS, GÊNEROS E/OU ESPÉCIES NÃO CATALOGADAS EM VICENTE ET AL. (1985)}

\section{Trichinelloidea}

\section{Trichuridae Railliet, 1915}

\section{Trichuridae sp.}

Hospedeiros: Schizodon fasciatus Agassiz, Pseudoplatistoma coruscans (Agassiz).

Localização: intestino.

Proveniência: rio Paraná, Guaíra, Paraná.

Referência bibliográfica: MORAVEC et al. (1992b).

\section{Capillaria Zeder, 1800}

\section{Capillaria sp.}

Hospedeiro: Plecostomus derbyi Haseman, 1911.

Localização: intestino.

Proveniência: Salto Osório, Salto Santiago, rio Iguaçu, Paraná

Referência bibliográfica: KoHN et al. (1988).

Comentário. As espécies catalogadas anteriormente (VICENTE et al. 1985) na super-

familia Trichuroidea foram incluidas em Trichinelloidea (ANDERSON \& BAIN 1982). 


\section{Dioctophymatoidea}

\section{Dioctophymatidae Railliet, 1915}

\section{Eustrongylides Jägerskiöld, 1909}

\section{Eustrongylides ignotus Jägerskiöld, 1909 (Figs 1-3)}

Hospedeiros: Auchenipterus nigripinnis (Boulanger), Brycon hilarii (Val.), Galeocharax humeralis (Val.), Hoplias malabaricus (Bloch), Paratrigon sp., Pinirampus pinirampu (Spix), Poecilidae sp., Pseudoplatystoma corruscans (Agassiz), Pseudoplatystoma fasciatus (L.), Raphiodon vulpinus (Agassiz), Salminus maxillosus (Cuv. e Val.), Serrasalmus nattereri (Kner), Symbranchus marmoratus (Bloch).

Localização: musculatura.

Proveniência: Porto Jofre, Porto Caracará, rio Cuiabá, Mato Grosso; Cachimbo, Pará.

Referências bibliográficas: EIRAS \& RÊGO (1988), RÊGO \& EIRAS (1988), RÊGO \& VICENTE (1988).

\section{Oxyuroidea}

\section{Pharyngodonidae Travassos, 1919}

Boca com seis lamelas. Cutícula espessada, com estriações transversais distintas. Macho com cauda truncada, com papilas caudais, apresentando um espículo e raramente com gubernáculo, formações esclerotizadas e apêndice caudal delgado e longo, frequentemente presentes. Parasitos de peixes, anfíbios e répteis.

Gênero tipo: Pharyngodon Diesing, 1861

\section{Brasilnema Moravec, Kohn \& Fernandes, 1992}

Corpo pequeno, com estriação transversal distinta; asas laterais presentes em ambos os sexos. Abertura oral hexagonal, sem lábios, rodeada por seis lamelas triangulares. Cápsula bucal larga. Cauda com apêndice terminal muito longo em ambos os sexos. Macho: gubernáculo ausente; espículo simples, moderadamente esclerotizado; papilas genitais claramente separadas em um grupo anterior ao cone genital protuso e outro par posterior ao mesmo. Asas caudais ausentes. Fêmea: vulva pré-equatorial; didelfa, ovos larvados, providos de longos filamentos polares. Parasitos de peixes de água doce.

Espécie tipo: B. pimelodellae Moravec, Kohn \& Fernandes, 1992

Brasilnema pimelodellae Moravec, Kohn \& Fernandes, 1992 (Figs 16-20, Tab. I)

Hospedeiro: Pimelodella lateristriga (Muller \& Troschel), "mandi-chorão".

Local de infecção: intestino.

Proveniência: Guaíra, rio Paraná, Paraná.

Referências bibliográficas: MORAVEc et al. (1992a,b).

Parasynodontisia Moravec, Kohn \& Fernandes, 1992

Corpo pequeno, com cutícula finamente estriada transversalmente; asas laterais ausentes. Abertura oral triangular, sem lábios, rodeada por seis lamelas triangulares. Cápsula bucal ausente. Cauda cônica em ambos os sexos. Macho: gubernáculo ausente; espículo simples, moderadamente esclerotizado; papilas genitais claramente separadas em um grupo anterior ao cone genital protuso e outro par posterior ao mesmo; asas caudais ausentes. Fêmea: vulva pré-equatorial; anfidelfa, ovos larvados, sem filamentos polares. Parasitos de peixes de água doce.

Espécie tipo: P. petterae Moravec, Kohn \& Fernandes, 1992 
Parasynodontisia petterae Moravec, Kohn \& Fernandes, 1992 (Figs 21-24, Tab. I)

Hospedeiro: Rhinelepis aspera Spix, "cascudo preto".

Localização: intestino.

Proveniência: Guaíra, rio Paraná, Paraná.

Referências bibliográficas: MORAVEC et al. (1992a,b).

Ichthyouris Inglis, 1962

Abertura oral hexagonal, circundada por seis pequenos lobos labiais; anfídeos pedunculados; parte anterior do esôfago extremamente modificada nas fềmeas; asas laterais presentes em ambos os sexos; cauda com espinho terminal muito longo em ambos os sexos. Macho: sem gubernáculo; asas caudais contínuas com as asas laterais de corpo e não suportadas pelas papilas caudais; dois pares de papilas longas e pedunculadas; um par de estruturas em forma de placas esclerotizadas, localizadas lateral e posteriormente à abertura cloacal.

Espécie tipo: I. ro Inglis, 1962

Ichthyouris brasiliensis Moravec, Kohn \& Fernandes, 1992 (Figs 4-7, Tab. I)

Hospedeiro: Pterygoplichthys aculeatus (Perugia), "cascudo abacaxi”.

Localização: intestino.

Proveniência: Guaíra, rio Paraná, Paraná.

Referências bibliográficas: INGLIS (1962), MORAVEC et al. (1992a,b).

Ichthyouris laterifilamenta Moravec, Kohn \& Fernandes, 1992 (Figs 8-11, Tab. I)

Hospedeiro: Trachydoras paraguyaensis (Eigenmann e Ward) "armadinho".

Localização: intestino.

Proveniência: Guaíra, rio Paraná, Paraná.

Referência bibliográfica: MORAVEC et al. (1992a).

Travnema Pereira, 1938

Travnema araujoi Fernandes, Campos \& Artigas, 1983 (Figs 12-15, Tab. I) Steind.

Hospedeiros: Curimatus gilberti Quoy \& Gaimard, Pseudocurimata elegans elegans

Localização: intestino.

Proveniência: Represa de Ilha Solteira, São Paulo e rio Paraná, Paraná.

Referências bibliográficas: FERNANDES et al. (1983), MORAVEC et al. (1992a).

\section{Seuratoidea}

\section{Quimperiidae Baylis, 1930}

Lábios pequenos ou ausentes. Sem cápsula bucal. Esôfago dividido ou não. Ventrículo e divertículo intestinal ausentes. Macho: ventosa pré-cloacal usualmente presente, sem anel esclerotizado. Espículos iguais, gubernáculo presente ou ausente. Fêmeas: vulva na metade posterior do corpo. Didelfas, anfidelfas. Ovíparas. Parasitos de peixes, ocasionalmente de anfíbios.

Gênero tipo: Quimperia Gendre, 1926 


\section{Neoparaseuratum Moravec, Kohn \& Fernandes, 1992}

Extremidade arredondada; boca hexagonal rodeada por seis cristas que se originam da margem bucal; quatro papilas cefálicas submedianas e dois anfídeos laterais presentes. Esôfago longo, não dividido, posteriormente mais largo; três pequenos dentes esofageanos presentes. Região cefálica do corpo rodeada por numerosas bandas longitudinais de cutícula inflada. Deirídeos pequenos, ao nível do esôfago. Poro excretor pós-esofageano. Macho: asas caudais ausentes, bandas musculares obliquas, presentes na região pré-cloacal. Espículos iguais, gubernáculo curto presente. Fêmea: vulva pós-equatorial, anfidelfa. Ovos não embrionados "in utero". Parasitos de intestino de peixes de água doce da América do Sul.

Espécie tipo: N. travassossi Moravec, Kohn \& Fernandes, 1992

Neoparaseuratum travassossi Moravec, Kohn \& Fernandes, 1992 (Figs 43-45, Tab. III)

Hospedeiros: Pterodoras granulosus (Val.), “armado, mandi-capeta”, Trachydoras paraguayensis (Eigenmann) "armadinho".

Localização: intestino.

Proveniência: rio Paraná, Paraná.

Referências bibliográficas: MORAVEc et al. (1992c, 1993a).

Seuratoidea sp. (Figs 95-97, Tab. VII)

Hospedeiro: Paulicea luetkeni (Steindachner).

Localização: estômago.

Proveniência: rio Paraná, Guaíra, Paraná.

Referência bibliográfica: MORAVEC et al. (1997).

Cucullanidae Cobbold, 1864

Cucullanus Mueller, 1777

Cucullanus brevispiculus Moravec, Kohn \& Fernandes, 1993 (Figs 25-27, Tab. II)

Hospedeiro: Auchenipterus nuchalis (Spix) "surumanha".

Localização: intestino.

Proveniência: Itaipu, Foz do Iguaçu, Paraná.

Referência bibliográfica: MORAVEC et al. (1993a).

Cucullanus cassinensis Pereira \& Costa, 1996 (Figs 90-92, Tab. VIII)

Hospedeiro: Micropogonias furnieri (Desmarest, 1823).

Localização: aparelho digestivo.

Proveniência: Lagoa dos Patos, Rio Grande do Sul.

Referência bibliográfica: PEREIRA \& COSTA (1996).

Cucullanus debacoi Sarmento, Fortes \& Hoffmann, 1995

Hospedeiro: Pimelodus maculatus Lacépède.

Localização: intestino.

Proveniência: rio Guaiba, Porto Alegre, Rio Grande do Sul.

Referência bibliográfica: SARMENTO et al. (1995).

Cucullanus fabregasi Fortes, Hoffmann, Sarmento, 1993 (Figs 83-84, Tab. VIII)

Hospedeiro: Pimelodus maculatus Lacépède, 1803.

Localização: intestino.

Revta bras. Zool. 16 (3): 561 - 610, 1999 
Proveniência: rio Guaiba, Porto Alegre, Rio Grande do Sul.

Referência bibliográfica: ForTES et al. (1993b).

Cucullanus grandistomis (Ferraz \& Thatcher, 1988) Moravec, Kohn \& Fernandes, 1993 (Figs 28-29, Tab. II)

Sinonímia: Bacudacnitis grandistomis Ferraz \& Thatcher, 1988.

Hospedeiro: Pseudoras niger (Val.).

Localização: intestino.

Proveniência: rio Amazonas, Amazonas.

Referências bibliográficas: FERRAz \& THATCHER (1988), MORAVEC et al. (1993a).

Cucullanus patoi Fortes, Hoffmann \& Sarmento, 1992 (Figs 30-31, Tab. II)

Hospedeiro: Pimelodus maculatus Lacépède, 1803.

Localização: intestino.

Proveniência: Lago do Guaíba, Rio Grande do Sul.

Referência bibliográfica: FORTES et al. (1992).

Cucullanus pimelodellae Moravec, Kohn \& Fernandes, 1993 (Figs 32-34, Tab. II)

Hospedeiro: Pimelodella lateristriga (Mueller \& Troschel) "mandi-chorão".

Localização: intestino.

Proveniência: rio Paraná Paraná.

Referência bibliográfica: MORAVEC et al. (1992a).

Cucullanus pinai pterodorasi Moravec, Kohn \& Fernandes, 1997 (Figs 98-100, Tab. VII)

Hospedeiro: Pterodoras gramulosus (Valenciennes).

Localização: intestino.

Proveniência: Reservatório de Itaipu, Guaíra, Paraná.

Referência bibliográfica: MoRA VEC et al. (1997).

Cucullanus pseudoplatystomae Moravec, Kohn \& Fernandes, 1993 (Figs 37-38, Tab. II)

Hospedeiro: Pseudoplatystoma coruscans (Agassiz) "pintado".

Localização: intestino.

Proveniência: rio Paraná, Paraná.

Referência bibliográfica: MORAVEC et al. (1993a).

Cucullanus rhamphichthydis Moravec, Kohn \& Fernandes, 1997 (Figs 101-102, Tab. VII)

Hospedeiro: Rhamphichthys rostratus (Linnaeus).

Localização: intestino.

Proveniência: Reservatório de Itaipu, Sta Helena, Paraná.

Referência bibliográfica: MORAVEC et al. (1997).

Cucullanus riograndensis Fortes, Hoffmann \& Sarmento, 1992 (Figs 35-36, Tab. III)

Hospedeiro: Pimelodus maculatus Lacépède, 1803.

Localização: intestino. 
Proveniência: Lago do Guaíba, Rio Grande do Sul.

Referência bibliográfica: ForTES et al. (1993a).

\section{Cucullanus sp.} "armado".

Hospedeiros: Paulicea luetkeni (Steindachner), “jaú", Pterodoras granulosus (Val.),

Local de infecção: intestino.

Proveniência: Foz do Iguaçu, Rio Paraná, Paraná.

Referência bibliográfica: MORAVEC et al. (1993a).

\section{Dichelyne Jägerskiöld, 1902}

Boca perpendicular ao eixo do corpo ou ligeiramente inclinada; placas da pseudo cápsula bucal pouco numerosas e separadas por uma simples sutura em forma de $\mathrm{Y}$; boca sustentada por uma estrutura peribucal esclerotizada com as peças esclerotizadas que se projetam dos músculos subjacentes do esôfago. Ceco intestinal presente. Machos: espículos iguais ou subiguais, gubernáculo presente em forma de Y. Ventosa pré-cloacal presente ou ausente. Fêmeas: vulva pós-equatorial. Parasitos de peixes e quelônios.

Espécie tipo: D. fossor Jägerskiöld, 1902

Dichelyne (C.) amaruincai (Freitas, Vicente \& Ibanez, 1969) Petter, 1974 (Figs 85-87, Tab. VIII)

Hospedeiro: Micropogonias furnieri (Desmarest).

Localização: aparelho digestivo.

Proveniência: Lagoa dos Patos.

Referência bibliográfica: PERLiRA \& COSTA (1996).

Dichelyne (C.) elongatus (Törnquist, 1931) Petter, 1974 (Figs 39-40, Tab. III)

Hospedeiros: Paralonchurus brasiliensis (Steind.) "maria-luiza" e Micropogonias furnieri (Desmarest).

Localização: intestino.

Proveniência: Ilha de Marambaia, Rio de Janeiro e Lagoa dos Patos, Rio Grande do Sul. (1991).

Referências bibliográficas: PEREIRA \& Cost^ (1996), Pinto et al. (1992), THATCHER

Dichelyne leporini Petter, 1989 (Figs 41-42, Tab. III)

Hospedeiro: Schizodon fasciatus Agassiz, "piau”.

Localização: intestino.

Proveniência: rio Paraná, Paraná.

Referência bibliográfica: MORAVEC et al. (1993a).

Dichelyne pimelodi Moravec, Kohn \& Fernandes, 1997 (Figs 103-105, Tab. VII)

Hospedeiro: Pimelodus maculatus (Lacépède).

Localização: intestino.

Proveniência: Reservatório de Itaipu, Guaíra, Paraná.

Referência bibliográfica: MORAVEC et al. (1997). 
Dichelyne (D.) micropogonii Pereira \& Costa, 1996 (Figs 88-89, Tab. VIII)

Hospedeiro: Micropogonias furnieri (Desmarest, 1823).

Localização: aparelho digestivo.

Proveniência: Lagoa dos Patos, Rio Grande do Sul.

Referência bibliográfica: PEREIRA \& COSTA (1996).

Dichelyne (Cucullanellus) sp.

Hospedeiro: Paralonchurus brasiliensis (Steind.).

Localização: intestino.

Proveniência: Ilha de Marambaia, Rio de Janeiro.

Referência bibliográfica: PINTo et al. (1992).

Comentário: duas outras espécies brasileiras de Dichelyne: D. (Cucullanellus) rodriguesi (Pinto, Fábio \& Noronha, 1970) Petter, 1974 e D. (C.) travassosi (Guimarães \& Cristófaro, 1974) Vicente, Pinto \& Aguilera, 1989, foram referidas anteriormente (VICENTE et al. 1985) como Cucullanellus rodriguesi Pinto, Fábio \& Noronha, 1970 e Cucullanellus travassosi Guimarães \& Cristófaro, 1974, respectivamente.

As espécies dos gêneros Cucullanus, Cucullanellus, Camallanus e Procamallamus foram referidas em Spiruroidea Railliet \& Henry, 1915, por Vicente et al. (1985).

Seuratoidea $\mathrm{sp}$.

Hospedeiro: Paulicea luetkeni (Steindachner).

Localização: estômago.

Proveniência: rio Paraná, Guaíra, Paraná.

Referência bibliográfica: MORAVEC et al. (1997).

\section{Cosmocercoidea}

\section{Cosmocercidae Travassos, 1925}

Spectatus sp. (Figs 93-94, Tab. VII)

Hospedeiro: Piaractus mesopotamicus (Holmberg).

Localização: intestino.

Proveniência: rio Paraná, Foz do Iguaçu, Paraná.

Referência bibliográfica: MORAVEC et al. (1997).

Ascaridoidea

Anisakidae Skrjabin \& Karokhin, 1945

Raphidascaris Railliet \& Henry, 1915

Raphidascaris (S.) hypostomi (Petter \& Cassone, 1984) Moravec, Kohn \& Fernandes, 1990 (Figs 46-47, Tab. IV)

Sinonímia: Sprentascaris hypostomi Petter \& Cassone, 1984.

Hospedeiros: Plecostomus albopunctatus Regan, P. commersoni (Val.), P. derbyi Haseman, Ancistrus cirrosus (Val.).

Localização: intestino e estômago.

Proveniência: Salto Osório, Paraná e Passo Fundo, Rio Grande do Sul.

Referência bibliográfica: MORAVEC et al. (1990). 
Raphidascaris (S.) mahnerti (Petter \& Cassone, 1984) Moravec, Kohn \& Fernandes, 1990 (Figs 48-50, Tab. IV)

Sinonímia: Sprentascaris mahnerti Petter \& Cassone, 1984.

Hospedeiros: Loricaria laticeps Regan, Loricariichthys sp..

Localização: intestino.

Proveniência: rio Paraná, Guaíra, Paraná.

Referências bibliográficas: MORAVEC et al. (1990, 1993a).

Raphidascaris (S.) sp.

Hospedeiro: Geophagus brasiliensis (Quoy \& Gaimard, 1842).

Localização: intestino.

Proveniência: rio Paraná, Paraná.

Referência bibliográfica: MoRAVEC et al. (1993a).

\section{Raphidascaris sp.}

Hospedeiro: Pagrus pagrus $\mathrm{L}$.

Localização: cavidade geral e intestino.

Proveniência: Rio de Janeiro, Rio de Janeiro.

Referência bibliográfica: RÊGO et al. (1985).

\section{Raphidascaris sp.}

Hospedeiros: Plecostomus albopunctatus Regan, 1908; P. derbyi Haseman, 1911.

Localização: intestino.

Proveniência: bacia do rio Iguaçu, Paraná.

Referência bibliográfica: KoHN et al. (1988).

\section{Raphidascaroides Yamaguti, 1941}

Lábios com fileiras de dentículos e expansões cuticulares laterais; interlábios presentes. Esôfago musculo-glandular seguido por um ventrículo glandular, do qual parte um apêndice posterior. Ceco intestinal ausente. Poro excretor posterior ao anel nervoso. Macho com cauda cônica e com sua extremidade coberta por numerosos espinhos rudimentares. Numerosas papilas pré-cloacais e poucas pós-cloacais; espículos iguais, alados; gubernáculo ausente. Testículo originando-se um pouco atrás do esôfago. Fêmea com cauda cônica, apresentando toda a sua extremidade coberta por minúsculos espinhos. Vulva situada no meio do corpo; ovários originando-se na parte posterior do corpo; úteros na região mediana do corpo. Ovos subglobulares, de casca fina, não segmentados. Parasitos de peixes teleósteos.

Espécie tipo: R. nipponensis Yamaguti, 1941.

\section{Raphidascaroides brasiliensis Moravec \& Thatcher, 1997 (Figs 106-109)}

Hospedeiro: Pterodoras granulosus (Valenciennes).

Localização: intestino.

Proveniência: lago Januacá, rio Amazonas, Manaus, Amazonas.

Referência bibliográfica: MORAVEC \& THATCHER (1997b).

Goezia Zeder, 1800

Goezia brasiliensis Moravec, Kohn \& Fernandes, 1994 (Figs 77-79, Tab. VI)

Hospedeiros: Brycon hilarii (Val.), Pseudoplatistoma corruscans (Agassiz).

Localização: intestino. 
Proveniência: rio Paraná, Foz do Iguaçu, Paraná.

Referência bibliográfica: MORAVEC et al. (1994a).

Goezia brevicaeca Moravec, Kohn \& Fernandes, 1994 (Figs 80-82, Tab. VI)

Hospedeiro: Brycon hilarii (Val.).

Local de infecção: estômago.

Proveniência: rio Paraná, Foz do Iguaçu, Paraná.

Referências bibliográficas: MORAvEc et al. (1993c), VicENTE et al. (1985).

Goezia sp.

Hospedeiros: Raphiodon vulpinus Agassiz, Serrasalmus marginatus (Val.), Ageneiosus valenciennesi Bleeber.

Localização: intestino.

Proveniência: Rio Paraná, Paraná.

Referência bibliográfica: MORAVEC et al. (1993a).

Hysterothylacium Ward \& Magath, 1917

Hysterothylacium $\mathrm{sp}$.

Hospedeiro: Pagrus pagrus L..

Localização: cavidade geral e intestino.

Proveniência: Rio de Janeiro, Rio de Janeiro.

Referência bibliográfica: RÊGo et al. (1985).

Hysterothylacium $\mathrm{sp}$.

Hospedeiros: Raphiodon vulpinus Agassiz, Salminus maxillosus Val., Galeocharax knerii (Steind.), Hypophthalmus edentatus Spix, Pterodoras granulosus (Val.), Loricariichthys sp., Plagioscium squamosissimus (Heckel), Crenicichla lepidota Heckel.

Localização: no intestino, livres ou encistados.

Proveniência: rio Paraná, Paraná.

Referência bibliográfica: MORAVEC et al. (1993a).

\section{Contracaecum Railliet \& Henry, 1912}

\section{Contracaecum sp.}

Hospedeiros: Acestrorhamphus macrolepis (Steind., 1876), Bergiaria sp., Crenicichla lepidota Heckel, 1840, Geophagus brasiliensis (Quoy e Gaimard, 1842), Hoplias malabaricus malabaricus (Block, 1794), Pimelodus ortmani Hasemann.

Localizaçao: estômago e peritôneo.

Proveniência: bacia do rio Iguaçu, Paraná.

Referência bibliográfica: KoHN et al. (1988).

\section{Contracaecum sp.}

Hospedeiros: Galeocharax humeralis Val., Galeocharax knerii (Steind.), Raphiodon vulpinus (Agassiz), Hoplias malabaricus malabaricus Bloch, Plagioscion squamosissimus Heckel, Crenicichla lepidota Heckel, Prochilodus scrofa Steind., Pseudoplatystoma coruscans (Agassiz). livre.

Localização: cavidade abdominal, peritôneo, mesonefro, estômago, encistado ou

Proveniencia: rio Paraná, Paraná.

Referência bibliográfica: MORAVEC et al. (1993a). 
Terranova Leiper \& Atkinson, 1914

Terranova sp.

Hospedeiro: Pagrus pagrus L.

Localização: cavidade geral e intestino.

Proveniência: Rio de Janeiro, Rio de Janeiro.

Referência bibliográfica: RÊGO et al. (1985).

Ascarididae Baird, 1853

Amplicaecum Baylis, 1920

Amplicaecum sp.

Hospedeiros: Hoplias malabaricus (Block), Leporinus copelandii Steind., 1875.

Localização: intestino.

Proveniência: rio Mogi-Guaçú, São Paulo.

Referência bibliográfica: KoHN \& FERNANDES (1987).

\section{Camallanoidea}

Camallanidae Railliet \& Henry, 1915

Camallanus Railliet \& Henry, 1915

Camallanus acaudatus Ferraz \& Thatcher, 1990 (Figs 51-54, Tab. IV)

Hospedeiro: Osteoglossum bicirrhosum.

Localização: intestino.

Proveniência: rio Amazonas, Amazonas.

Referência bibliográfica: FERRAZ \& THATCHER (1990).

Camallanus tridentatus (Drasche, 1884) Railliet \& Henry, 1915 (Figs 55-56, Tab. IV)

Hospedeiro: Osteoglossum bicirrhosum Vandelli, 1829

Localização: intestino.

Proveniência: rio Amazonas, Amazonas.

Referência bibliográfica: FERRAZ \& THATCHER (1990).

Comentário: somente fềmeas dessa espécie haviam sido referidas anteriormente (VICENTE et al. 1985).

\section{Oncophora Diesing, 1851}

Corpo alongado, afilando-se nas extremidades. Cápsula bucal com cristas longitudinais numerosas e denteadas anteriormente e curtas, pouco numerosas, posteriormente. Tridentes presentes, com comprimento alongado. Fêmeas adultas com brusco alargamento anterior à vulva. Fêmeas jovens sem essa característica, mas apresentando vulva proeminente circundada por uma dobra elevada e fortemente esclerotizada. Machos com dois espículos, um dos quais inconspícuo, às vezes de difícil observação. Gubernáculo ausente. Sete pares de papilas pré-cloacais. Parasitos de peixes marinhos.

Espécie tipo e única: O. melanocephala (Rud., 1819) Baudin-Laurencin, 1971. 
Oncophora melanocephala (Rudolphi, 1819) Baudin-Laurencin, 1971 (Figs 57-59, Tab. IV)

Hospedeiro: Priacanthus arenatus Cuvier, 1829, "olho-de-cão".

Localização: intestino.

Proveniência: Cabo Frio, Rio de Janeiro.

Referência bibliográfica: PINTO et al. (1988).

\section{Paracamallanus Yorke \& Maplestone, 1926}

Boca formada por duas valvas esclerotizadas, com espessamentos longitudinais em forma de costelas e denteação marginal. Processo esclerotizado em forma de tridente na junção das valvas, em ambos os lados; ampla cavidade bucal esclerotizada entre as valvas e o esôfago. Parasitos de peixes de água doce.

Espécie tipo: P. cyathopharyne (Baylis, 1923) Yorke \& Maplestone, 1926

Paracamallanus amazonensis Ferraz \& Thatcher, 1992 (Figs 60-62, Tab. V)

Hospedeiros: Hypophthalmus edentatus Spix, Pterodoras granulosus (Val.).

Localização: intestino.

Proveniência: rio Amazonas, Amazonas e rio Paraná, Paraná.

Referências bibliográficas: FERRAZ \& THATCHER (1992), MORAVEC et al. (1993b).

Paracamallanus sp.

Hospedeiro: Hypophthalmus edentatus Spix.

Localização: intestino.

Proveniência: rio Amazonas, Amazonas.

Referência bibliográfica: THATCHER (1991).

\section{Procamallanus Baylis, 1923}

Procamallanus (Procamallanus) annipetterae (Kohn \& Fernandes, 1988) Kohn \& Fernandes, 1988 (Figs 63-65, Tab. V)

Sinonímia: Procamallanus petterae Kohn \& Fernandes, 1988, nec Procamallamus (Procamallanus) petterae Moravec \& Sey, 1988.

Hospedeiro: Plecostomus albopunctatus Regan, 1908, "cascudo-ferro".

Localização: intestino.

Proveniência: rio Iguaçu, Paraná.

Referências bibliográficas: KoIn \& FERNANDES (1988a,b), MORAVEC \& SEY (1988), PETTER (1990), Rodrigues et al. (1991).

Procamallanus (Spirocamallanus) freitasi (Moreira, Oliveira \& Costa, 1991) (Figs 66-67, Tab. V)

Sinonímia: Spirocamallanus freitasi Moreira, Oliveira \& Costa, 1991.

Hospedeiros: Bergiaria westermanni (Reinhardt, 1874), Pimelodus maculatus Lacépède, 1803, Pimelodus sp..

Localização: intestino.

Proveniência: Rio São Francisco, Minas Gerais.

Referências bibliográficas: MOREIRA et al. (1991), RodRIGUES et al. (1991). 
Procamallanus (Spirocamallanus) pintoi (Kohn \& Fernandes, 1988) Rodrigues, Pinto \& Noronha, 1991 (Figs 68-70, Tab. V)

Sinonímia: Spirocamallanus pintoi Kohn \& Fernandes, 1988.

Hospedeiro: Corydoras paleatus (Genyns, 1842) "cascudinho".

Localização: intestino.

Proveniência: rio Iguaçu, Paraná.

Referências bibliográficas: KOHN \& FERNANDES (1988a), RODRIGUES et al. (1991).

Procamallanus (Spirocamallanus) saofranciscencis (Moreira, Oliveira \& Costa, 1994) comb.n. nhardt).

Hospedeiros: Tetragonopterus chalceus Agazzis e Acestorhinchus lacustris (Rei-

Localização: cavidade celomática, estômago, intestino, ceco pilórico.

Proveniência: Represa de Tres Marias, rio São Francisco, Minas Gerais.

Referência bibliográfica: Moreira et al. (1991).

\section{Procamallanus sp.}

Hospedeiros: Salminus hilarii Valenciennes, 1849, "tabarana"; Prochilodus scrofa Steind., "curimbata".

Localização: intestino.

Proveniência: rio Mogi-Guaçú, São Paulo.

Referências bibliográficas: KoHN \& FERNANDES (1987), RodRIGUES et al. (1991).

\section{Procamallanus sp.}

Hospedeiro: Astianax fasciatus fasciatus (Cuvier, 1819).

Localização: intestino.

Proveniência: bacia do rio Iguaçu, Paraná.

Referências bibliográficas: KoHN et al. (1988), RodRIGUES et al. (1991).

Comentário: Cucullanidae e Camallanidae foram anteriormente incluídas em Spiruroidea Railliet \& Henry, 1915.

\section{Acuarioidea}

\section{Acuariidae Railliet, Henry \& Sisoff, 1912}

Nematóides com dois lábios laterais. Geralmente providos de cordões ou apêndices cuticulares na parte anterior do corpo. Vestíbulo longo e cilíndrico. Esôfago cilíndrico dividido em duas partes. Machos com asas caudais e papilas pedunculadas. Espículos desiguais e dissemelhantes. Fêmeas geralmente com ovejetor e vagina curtos. Parasitos de aves, répteis, mamíferos e, ocasionalmente de peixes que funcionam como hospedeiros paratênicos.

Gênero tipo: Acuaria Bremser, 1811.

\section{Acuariinae Railliet, Henry \& Sisoff, 1912}

Com as características da familia.

Acuariinae sp.

Hospedeiro: Salminus maxillosus Val..

Localização: estômago.

Proveniência: rio Paraná, Paraná.

Referência bibliográfica: MORAVEC et al. (1993a). 


\title{
Dracunculoidea
}

\author{
Guyanemidae Petter, 1974
}

\section{Guyanema Petter, 1974}

Extremidade apical com quatro papilas cefálicas, quatro papilas labiais externas e quatro internas. Tanto as papilas cefálicas quanto as labiais são deslocadas lateralmente. Ausência de cápsula bucal esclerotizada. Esôfago dividido em curta porção muscular e longa porção glandular. Cauda da fêmea longa. Vulva situada na porção anterior do corpo. Machos com espículos iguais. Cauda longa com asas caudais. Vivíparos. Parasitos de peixes.

Espécie tipo: G. seriei Petter, 1974.

Guyanema raphidioni Moravec, Kohn \& Fernandes, 1993 (Figs 71-72, Tab. V)

Hospedeiro: Raphiodon vulpinus Agassiz, "dourado cachorro".

Localização: intestino.

Proveniência: Itaipu, Paraná.

Referências bibliográficas: MoRAvec et al. (1993b,c), PETTER (1974a).

\section{Guyanema sp.}

Hospedeiro: Pseudocurimata g. gilberti (Quoy \& Gaimard) "sairú”).

Localização: intestino.

Proveniência: Itaipu, Paraná.

Referência bibliográfica: MORAVEC et al. (1993b).

Comentário: as características genéricas são as mesmas utilizadas para a diagnose da família.

\section{Travassosnema Costa, Moreira \& Oliveira, 1991}

Cápsula bucal ausente. Esôfago dividido em duas porções, uma muscular e outra glandular, sendo que a última com apêndice posterior livre, próximo à junção com o intestino. Ânus funcional. Vulva anterior, desenvolvida em fêmeas maduras. Monodelfas. Asas laterais bem desenvolvidas nos machos. Parasitos de peixes.

Espécie tipo: T. travassosi Costa, Moreira \& Oliveira, 1991

Travassosnema travassosi Costa, Moreira \& Oliveira, 1991 (Fig. 73, Tab. $\mathrm{VI})$

Hospedeiro: Acestrorhynchus lacustris Reinhardt, 1874.

Localização: tecidos atrás dos olhos.

Proveniência: Reservatório de Três Marias, Minas Gerais.

Referência bibliográfica: CosT^ et al. (1991).

Travassosnema travassosi paranaensis Moravec, Kohn \& Fernandes, 1993 (Figs 74-76, Tab. VI)

Hospedeiro: Acestrorhynchus lacustris Reinhardt, 1874 ("peixe-cachorro").

Localização: cavidade do corpo.

Proveniência: rio Paraná, Paraná.

Referência bibliográfica: MORAVEC et al. (1993c). 


\title{
ESPÉCIES CATALOGADAS (VICENTE ET AL. 1985) EM OUTROS HOSPEDEIROS
}

\author{
Cosmocercoidea
}

Atractidae Travassos, 1919

Klossinemella Costa, 1961

Klossinemella iheringi (Travassos, Artigas \& Pereira, 1928) Costa, 1961

Hospedeiro: Mylesimus paraschomburgkii Santos \& Ferreira, 1989 “jigu”.

Localização: intestino.

Proveniência: rio Trombetas e rio Jari, Pará; rios Uatuma, Pitinga e Capucapu, Amazonas e rio Araguari, Amapá.

Referências bibliográficas: ViCENTE et al. (1985), MORAVEC \& THATCHER (1997a).

Rondonia Travassos, 1919

Rondonia rondoni Travassos, 1919

Hospedeiro: Trachydoras paraguayensis (Eigenmann \& Ward) ("armadinho").

Localização: intestino.

Proveniência: rio Paraná, Paraná.

Referências bibliográficas: VICENTE et al. (1985), MORAVEC et al. (1992b).

Comentário: anteriormente (VICENTE et al. 1985) esta espécie havia sido incluída em Oxyuroidea Railliet, 1916.

\section{Seuratoidea}

\section{Cucullanidae Cobbold, 1864 \\ Cucullanus Mueller, 1777}

Cucullanus pulcherrimus Barreto, 1918

Hospedeiros: Paralonchurus brasiliensis (Steind.), Micropogonias furnieri (Desmarest).

Localização: intestino.

Proveniência: Ilha de Marambaia, Rio de Janeiro e Lagoa dos Patos, Rio Grande do Sul.

Referências bibliográficas: VICENTE et al. (1985), PINTO et al. (1992), PEREIRA \& Costa (1996).

Cucullanus pinnai pinnai Travassos, Artigas \& Pereira, 1928

Hospedeiros: Pseudopimelodus roosevelti Borodin, 1927 (= P. zungaro); Pimelodus ornatus Kner; P. maculatus (Lacépède); Ageneiosus valenciennesi Bleeker; Pimelodella gracilis (Valenciennes); Paulicea luetkeni (Steindachner); Loricaria sp.

Localização: intestino.

Proveniência: rio Mogi-Guaçú, São Paulo.

Referências bibliográficas: VICENTE et al. (1985), KOHN \& FERNANDES (1987), MORAVEC et al. (1993a).

Cucullanus zungaro Vaz \& Pereira, 1934

Hospedeiro: Pseudopimelodus zungaro (Humboldt).

Localização: intestino. 
Proveniência: foz do rio Iguaçu, Paraná.

Referência bibliográfica: VICENTE et al. (1985).

\section{Oxyuroidea}

\section{Pharyngodonidae Travassos, 1919 \\ Travnema Pereira, 1938}

\section{Travnema travnema Pereira, 1938}

Hospedeiros: Pseudocurimata elegans elegans (Steindachner) "sairu”, P. g. gilberti (Quoy \& Gaimard, 1824), Astyanax bimaculatus lacustris (Reinhardt).

Localização: intestino.

Proveniência: rio Paraná, Itaipu, Paraná e rio Mogi-Guaçú, São Paulo.

Referências bibliográficas: ViCENTE et al. (1985), KoHn \& FERNANDES (1987), MORAVEC et al. (1992b, 1994b).

Comentário: Esta espécie havia sido incluída (VICENTE et al. 1985) na família Oxyuridae.

$$
\text { Oxyuridae Cobbold, } 1864
$$

\section{Cosmoxynema Travassos, 1949}

Cosmoxynema vianai Travassos, 1949

Hospedeiros: Pseudocurimata elegans Steind.

Localização: intestino.

Proveniência: rio Paraná, Paraná.

Referência bibliográfica: VICENTE et al. (1985).

\section{Cosmoxynemoides Travassos, 1949}

Cosmoxynemoides aguirrei Travassos, 1949 inhardt).

Hospedeiros: Pseudocurimata elegans Steind., Astyanax bimaculatus lacustris (ReLocalização: intestino.

Proveniência: rio Paraná, Itaipu, Paraná.

Referências bibliográficas: ViCENTE et al. (1985), MORAVEC et al. (1994b).

Camallanoidea

Camallanidae Railliet \& Henry, 1915

Procamallanus Baylis, 1923

Procamallanus (S.) hilarii Vaz \& Pereira, 1934

Hospedeiro: Astianax bimaculatus schubarti Britski.

Localização: intestino.

Proveniência: rio Mogi Guaçú, São Paulo.

Referências bibliográficas: VicENTE et al. (1985), KOHN \& FERNANDES (1987), RÊGO \& VICENTE (1988).

Procamallanus (S.) iheringi Travassos, Artigas \& Pereira, 1928

Hospedeiro: Schizodon fasciatus Agassiz.

Localização: intestino. 
Proveniência: rio Paraná, Paraná.

Referências bibliográficas: VICENTE et al. (1985), RODRIGUES et al. (1991), MORAVEC et al. (1993b).

\section{Procamallanus (S.) inopinatus Travassos, Artigas \& Pereira, 1928}

Hospedeiros: Leporinus fasciatus (Bloch), Leporinus piau Fowler "piau-gordura", Leporinus reinhardti Lutken "piau-tres-pintas", Leporinus taeniatus Lutken "piau-jeju", Brycon erythropterum (Cope.), Mylopus asterias (Mueller \& Troschel, 1844), Salminus maxillosus (Val., 1849),Schizodon knerii (Steindachner) "piau-branco"Leporinus friderici (Bloch), Serrasalmus brandtii Reinhardt "pirambeba", Serrasalmus marginatus (Val.), Pterodoras gramulosus (Val.), Trachydoras paraguayensis (Eigenmann \& Ward), Crenicichla haroldoi (Luengo \& Britski), Potamotrygon motoro (Mueller \& Henle).

Localização: intestino.

Proveniência: rio Paraná, Manaus, Amazonas e rio Mogi-Guaçú, São Paulo.

Referências bibliográficas: VicENTE et al. (1985), KoHn \& FERNANDES (1987), Moravec et al. (1993b), Moreira et al. (1994), Rodrigues et al. (1991), THATCHER (1991).

Procamallanus (S.) pimelodus Pinto, Fábio, Noronha \& Rolas, 1974

Sinonímia: Procamallanus (S.) intermedius Pinto, Fábio, Noronha \& Rolas, 1974.

Hospedeiros: Pimelodella lateristriga (Mueller \& Troschel), Pimelodus ortmanni Hasemann, 1911.

Localização: intestino.

Proveniência: rio Paraná, Paraná.

Referências bibliográficas: Kolin et al. (1988), RoDRIGUES et al. (1991), MORAVEC et al. (1993b).

\section{Procamallanus (P.) peraccuratus Pinto, Fábio, Noronha \& Rolas, 1976}

Hospedeiros: Potamotrygon motoro (Mueller \& Henle), Pimelodus ortmanni Hasemann 1911, Crenicichla lepidota Heckel, 1840.

Localização: intestino.

Proveniência: rio Paraná, Paraná.

Referências bibliográficas: ViCENTE et al. (1985), KOHN et al. (1988), THATCHER (1991), MORAVEC et al. (1993b).

\section{LISTA ALFABÉTICA DOS HOSPEDEIROS E SEUS RESPECTIVOS NEMATÓIDES}

Acestrorhamphis macrolepis (Steindachner, 1876)

Contracaecum sp.

Acestrorhynchus lacustris Reinhardt, 1874

Procamallanus (S.) saofranciscencis (Moreira, Oliveira \& Costa, 1994) comb.n.

Travassosnema travassosi Costa, Moreira \& Oliveira, 1991

Travassosnema travassosi paranaensis Moravec, Kohn \& Fernandes, 1993

Ageneiosus valenciennesi Bleeker

Cucullanus pinnai Travassos, Artigas \& Pereira, 1928

Goezia sp.

Ancistrus cirrhosus (Valenciennes)

Raphidascaris (S.) hypostomi Petter \& Cassone, 1984

Astyanax bimaculatus lacustris (Reinhardt, 1874) 
Cosmoxynemoides aguirrei Travassos, 1949

Travnema travnema Pereira, 1938

Astyanax bimaculatus schubarti Britski, 1964

Procamallanus (S.) hilarii (Vaz \& Pereira, 1934)

Astianax fasciatus fasciatus (Cuvier)

Procamallanus sp.

Auchnipterus nigripinnis (Boulanger)

Eustrongylides ignotus Jägerskiöld, 1909

Auchenipterus nuchalis (Spix)

Cucullanus brevispiculus Moravec, Kohn \& Fernandes, 1993

Bergiaria sp.

Contracaecum sp.

Bergiaria westmanni (Reinhard)

Procamallanus (S.) freitasi (Moreira, Oliveira \& Costa, 1991) comb.n. Brycon erythropterum (Cope)

Procamallanus (S.) inopinatus Travassos, Artigas \& Pereira, 1928

Brycon hilarii (Valenciennes)

Eustrongylides ignotus Jägerskiöld, 1909

Goezia brasiliensis Moravec, Kohn \& Fernandes, 1994

Goezia brevicaeca Moravec, Kohn \& Fernandes, 1994

Corydoras paleatus

Procamallanus (S.) pintoi (Kohn \& Fernandes, 1988)

Crenicichla haroldoi (Luengo \& Britski)

Procamallanus (S.) inopinatus Travassos, Artigas \& Pereira, 1928

Crenicichla lepidota Heckel

Procamallanus (P.) peraccuratus Pinto, Fábio, Noronha \& Rolas, 1976

Contracaecum sp.

Curimatus gilberti Quoy \& Gaimard

Travnema araujoi Fernandes, Campos \& Artigas, 1983

Galeocharax humeralis Valenciennes

Contracaecum sp.

Eustrongylides ignotus Jägerskiöld, 1909

Galeocharax knerii (Steindachner)

Contracaecum sp.

Hysterothylacium sp.

Geophagus brasiliensis (Quoy \& Gaimard)

Contracaecum sp.

Hoplias malabaricus (Block)

Amplicaecum sp.

Contracaecum sp.

Eustrongylides ignotus Jägerskiöld, 1909

Hypophthalmus edentatus Spix

Hysterothylacium sp.

Paracamallanus amazonensis Ferraz \& Thatcher, 1992

Paracamallanus sp. 
Leporimus copelandii Steindachner

Amplicaecum sp.

Leporinus fasciatus (Bloch)

Procamallanus (S.) inopinatus Travassos, Artigas \& Pereira, 1928 Leporinus friderici (Bloch)

Procamallanus (S.) inopinatus Travassos, Artigas \& Pereira, 1928 Leporinus piau Fowler

Procamallanus (S.) inopinatus Travassos, Artigas \& Pereira, 1928 Leporinus reinhardti Lutken

Procamallanus (S.) inopinatus Travassos, Artigas \& Pereira, 1928 Leporimus taeniatus Lutken

Procamallanus (S.) inopinatus Travassos, Artigas \& Pereira, 1928 Loricaria laticeps

Raphidascaris (S.) mahnerti (Petter \& Cassone, 1984)

Loricaria sp.

Cucullamus pinai pinai Travassos, Artigas \& Pereira, 1928

Loricariichthys sp.

Hysterothylacium sp.

Raphidascaris (S.) mahnerti (Petter \& Cassone, 1984)

Micropogonias furnieri (Desmarest)

Dichelyne (C.) amaruincai (Freitas, Vicente \& Ibañez, 1969)

Dichelyne (C.) elongatus (Törnquist, 1931)

Dichelyne (D.) micropogonii Pereira \& Costa, 1996

Cucullanus cassinensis Pereira \& Costa, 1996

Cucullanus pulcherrimus Barreto, 1918

Mylesinus paraschomburgkii Santos \& Ferreira

Klossinemella iheringi (Travassos, Artigas \& Pereira, 1928)

Mylopus asterias (Mueller \& Troschel)

Procamallanus (S.) inopinatus Travassos, Artigas \& Pereira, 1928

Osteoglossum bicirrhosum

Camallanus acaudatus Ferraz \& Thatcher, 1990

Camallanus tridentatus (Drasche, 1884)

Pagrus pagrus Linnaeus

Hysterothylacium sp.

Raphidascaris sp.

Terranova sp.

Paralonchurus brasiliensis (Steindachner)

Cucullamus pulcherrimus Barreto, 1918

Dichelyne (C.) elongatus (Törnquist, 1931)

Dichelyne (C.) sp.

Paratrigon sp.

Eustrongylides ignotus Jägerskiöld, 1909

Paulicea luetkeni (Steindachner)

Cucullanus sp.

Revta bras. Zool. 16 (3): 561 - 610, 1999 
Cucullanus pinai pinai Travassos, Artigas \& Pereira, 1928

Seuratoidea sp.

Piaractus mesopotamicus (Holmberg) (= Mylossoma orbignyanus Valenciennes)

Spectatus sp.

Pimelodella gracilis (Valenciennes)

Cucullanus pinai pinai Travassos, Artigas \& Pereira, 1928

Pimelodella lateristriga (Muller \& Troschel)

Brasilnema pimelodellae Moravec, Kohn \& Fernandes, 1992

Cucullanus pimelodellae Moravec, Kohn \& Fernandes, 1992

Procamallanus (S.) pimelodus Pinto, Fábio, Noronha \& Rolas, 1974

Pimelodus sp.

Procamallanus (S.) freitasi (Moreira, Oliveira \& Costa, 1991)

Pimelodus maculatus Lacépède

Cucullanus debacoi Sarmento, Fortes \& Hoffmann, 1995

Cucullanus fabregasi Fortes, Hoffmann \& Sarmento, 1993

Cucullanus patoi Fortes, Hoffmann \& Sarmento, 1992

Cucullanus pinai pinai Travassos, Artigas \& Pereira, 1928

Cucullanus riograndensis Fortes, Hoffmann \& Sarmento, 1992

Dichelyne pimelodi Moravec, Kohn \& Fernandes, 1997

Procamallanus (S.) freitasi (Moreira, Oliveira \& Costa, 1991)

Pimelodus ornatus Kner

Cucullanus pinnai Travassos, Artigas \& Pereira, 1928

Pimelodus ortmanni Hasemann

Contracaecum sp.

Procamallanus (P.) peraccuratus Pinto, Fábio, Noronha \& Rolas, 1976

Procamallanus (S.) pimelodus (= intermedius) Pinto, Fábio, Noronha \& Rolas, 1974

Pinirampus pinirampu (Spix)

Eustrongylides ignotus Jägerskiöld, 1909

Plagioscium squamosissimus Heckel

Contracaecum sp.

Hysterothylacium sp.

Plecostomus albopunctatus

Procamallamus (S.) annipetterae (Kohn \& Fernandes, 1988)

Raphidascaris (S.) hypostomi (Petter \& Cassone, 1984)

Raphidascaris sp.

Plecostomus commersoni (Valenciennes)

Raphidascaris (S.) hypostomi (Petter \& Cassone, 1984)

Plecostomus derbyi Haseman

Capillariasp.

Raphidascaris (S.) hypostomi (Petter \& Cassone, 1984)

Raphidascaris sp.

Potamotrigon motoro (Mueller \& Henle)

Procamallanus (P.) peraccuratus Pinto, Fábio, Noronha \& Rolas, 1976

Procamallanus (S.) inopinatus Travassos, Artigas \& Pereira, 1928

Revta bras. Zool. 16 (3): 561 - 610, 1999 
Priacanthus arenatus Cuvier

Oncophora melanocephala (Rudolphi, 1819)

Prochilodus scrofa Steindachner

Contracaecum sp.

Procamallanus sp.

Psendocurimata elegans Steindachner

Cosmoxynema vianai Travassos, 1949

Cosmoxynemoides aguirrei Travassos, 1949

Travnema araujoi Fernandes, Campos \& Artigas, 1983

Travnema travnema Pereira, 1938

Pseudocurimata gilberti gilberti (Quoy \& Gaimard)

Guyanema sp.

Travnema travnema Pereira, 1938

Pseudopimelodus roosevelti Borodin, 1927

Cucullanus pinnai Travassos, Artigas \& Pereira, 1928

Pseudopimelodus zungaro (Humboldt)

Cucullanus zungaro Vaz \& Pereira, 1934

Pseudoplatistoma corruscans (Agassiz)

Contracaecum sp.

Cucullanus pseudoplatystomae Moravec, Kohn \& Fernandes, 1993

Eustrongylides ignotus Jägerskiöld, 1909

Goezia brasiliensis Moravec, Kohn \& Fernandes, 1994

Pseudoplatystoma fasciatus (Linnaeus)

Eustrongylides ignotus Jägerskiöld, 1909

Trichuridae sp.

Pseudoras niger (Valenciennes)

Cucullanus grandistomis (Ferraz \& Tatcher, 1988)

Pterodoras granulosus (Valenciennes)

Cucullanus pinai pterodorasi Moravec, Kohn \& Fernandes, 1997

Cucullanus sp.

Hysterothylacium sp.

Neoparaseuratum travassosi Moravec, Kohn \& Fernandes, 1992

Paracamallanus (S.) amazonensis Ferraz \& Tatcher, 1992

Procamallanus (S.) inopinatus Travassos, Artigas \& Pereira, 1928

Raphidascaroides brasiliensis Moravec \& Thatcher, 1997

Pterygoplichthys aculeatus

Ichthyouris brasiliensis Moravec, Kohn \& Fernandes, 1992

Raphiodon vulpinus (Agassiz)

Eustrongylides ignotus Jägerskiöld, 1909

Goezia sp.

Guyanema raphidioni Moravec, Kohn \& Fernandes, 1993

Hysterothylacium sp.

Rhinelepis aspera Spix

Parasynodontisia petterae Moravec, Kohn \& Fernandes, 1992

Revta bras. Zool. 16 (3): 561 - 610, 1999 
Salminus hilarii Valenciennes, 1849

Procamallanus sp.

Salminus maxillosus Cuvier \& Valenciennes

Acuariinae sp.

Eustrongylides ignotus Jägerskiöld, 1909

Hysterothylacium sp.

Procamallamus (S.) inopinatus Travassos, Artigas \& Pereira, 1928

Schizodon fasciatus Agassiz

Dichelyne leporini Petter, 1989

Procamallamus (S.) iheringi Travassos, Artigas \& Pereira, 1928

Trichuridae sp.

Schizodon knerii (Steindachner)

Procamallanus (S.) inopinatus Travassos, Artigas \& Pereira, 1928

Serrasalmus brandtii Reinhardt

Procamallanus (S.) inopinatus Travassos, Artigas \& Pereira, 1928

Serrasalmus marginatus (Valenciennes)

Goezia sp.

Procamallanus (S.) inopinatus Travassos, Artigas \& Pereira, 1928

Serrasalmus nattereri (Kner)

Eustrongylides ignotus Jägerskiöld, 1909

Symbranchus marmoratus (Bloch)

Eustrongylides ignotus Jägerskiöld, 1909

Tetragonopterus chalceus Agazzis

Procamallanus (Spirocamallanus) saofranciscensis (Moreira, Oliveira \& Costa, 1994) comb.n.

Trachydoras paraguayensis (Eigenmann \& Ward)

Ichthyouris laterifilamenta Moravec, Kohn \& Fernandes, 1992

Neoparaseuratum travassosi Moravec, Kohn \& Fernandes, 1992

Procamallanus (S.) inopinatus Travassos, Artigas \& Pereira, 1928

Rondonia rondoni Travassos, 1919

\section{REFERÊNCIAS BIBLIOGRÁFICAS}

ANDERSON, R.C. \& O. BAIN. 1982. Keys to genera of the Superfamilies Rhabditoidea, Dioctophymatoidea, Trichinelloidea and Muspiceoidea. In: R.C. ANDERSON; A.G. CHABAUD \& S. WILLMOTT (Eds). CIH Keys to the nematode parasites of vertebrates 9. Bucks, Commonwealth Agricultural Bureaux, Farnham Royal, 26p.

Chabaud, A.G. 1975a. Keys to genera of the order Spirurida. Part. I. Camallanoidea, Dracunculoidea, Gnathostomatoidea, Physalopteroidea, Rictularoidea and Thelazioidea. In: R.C. ANDERSON; A.G. ChaBAud \& S. WillmotT (Eds). CIH Keys to the nematode parasites of vertebrates 3. Parte I. Bucks, Commonwealth Agricultural Bureaux, Farnham Royal, 27p.

. 1975b. Keys to genera of the order Spirurida. Part. II. Spiruroidea, Camallanoidea, Habronematoidea and Acuarioidea. In: R.C. ANDERSON; A.G. CHABAUD \& S. WILLMOTT (Eds). CIH Keys to the nematode parasites of vertebrates 3. Bucks, Commonwealth Agricultural Bureaux, Farnham Royal, 29p.

. 1978. Keys to genera of the superfamily Cosmocercoidea, Seuratoidea, Heterakoidea and Subuluroidea. In: R.C. Anderson; A.G. Chabaud \& S. WillmotT 
(Eds). CIH Keys to the nematode parasites of vertebrates 6. Bucks, Commonwealth Agricultural Bureaux, Farnham Royal, 71p.

Costa, H.M.A.; M.P. Guimarães; D.N. Cabral \& M.J.S. Mundim. 1995. Scanning electron microscopic observations on Goezia spinulosa (Diesing, 1839) (Nematoda, Anisakidae) from Arapaima gigas (Cuvier, 1817). Mem. Inst. Oswaldo Cruz 90 (6): 703-705.

Costa, H.M.A.; N.I.B. Moreira \& C.L. Oliveira. 1991. Travassosnema gen.n. with the description of T. travassosi sp.n. (Dracunculoidea Guyanemidae) parasite of Acestrorhynchus lacustris Reinhardt, 1874 (Characidae) from Tres Marias Reservoir, MG, Brazil. Mem. Inst. Oswaldo Cruz 86: 437-439.

EIRAS, J.C. \& A.A. RÊGo. 1988. Histopatologia da parasitose de peixes do rio Cuiabá (Mato Grosso) por larvas de Eustrongylides sp. (Nematoda, Dioctophymidae). Rev. Brasil. Biol. 48: 273-280.

FERnANDES, M.T.; M.S. CAMPos \& P.T. ARTIGas. 1983. Travnema araujoi n.sp. (Nematoda, Pharyngodonidae) parasito de Curimatus gilberti Quoy \& Gaimard (Pisces, Characinidae) Rev. Fac. Med. vet. Zootec. Univ. São Paulo 20 (1): 35-41.

Ferraz, E.; V.E. Thatcher. 1988. Bacudacnitis grandistomis gen. et sp. nov. (Nematoda: Cucullanidae) parasita intestinal de um bagre, Psendoras niger (Valenciennes) da Amazônia brasileira. Amazoniana 10 (3): 249-250.

-1990. Camallanus acaudatus sp.n. (Nematoda, Camallanidae) e uma descrição do macho de Camallanus tridentatus (Drasche, 1884) parasitas de peixes da Amazonia brasileira. Amazoniana 11 (2): 135-145.

-1992. Paracamallamus amazonensis sp.n. (Nematoda, Camallanidae) um parasita de bagre, Hypophthalmus edentatus (Pisces: Hypophythalmidae) da Amazonia brasileira. Amazoniana 12: 1-6.

ForTES, E.; R.P. HoffMAnN \& T.M. SARMEnTo. 1992. Descrição de Cucullanus patoi sp.n. (Nematoda, Cucullanidae) de pintado Pimelodus maculatus Lacépède, 1803 (Pisces), do Lago do Guaíba, RS, Brasil. Ciênc. Rural 22 (3): 235-238.

- 1993a. Nova espécie de nematódeo Cucullanus, C. riograndensis sp.n. de Pimelodus maculatus Lacépède, 1803 do Lago do Guaíba, Rio Grande do Sul, Brasil. Rev. Bras. Med. Vet. 15 (3): 79-82.

1993b. Cucullanus fabregasi sp.n.: Nematoda parasita do intestino do peixe Pimelodus maculatus Lacépède, 1803 do Rio Guaíba, Porto Alegre, Rio Grande do Sul, Brasil. Arq. Fac. Vet. UFRGS, Porto Alegre, 21: 71-76.

INGLIS, W.G. 1962. Ichthyouris ro. gen. et sp. nov. (Nematoda): an oxyurid from a fresh water fish. Jour. Helminthol. 36: 45-50.

HaRTWICH, G. 1974. Keys to genera of the Ascaridoidea. In: R.C. ANDERson; A.G. CHABAUd \& S. WillmotT (Eds). CIH Keys to the nematode parasites of vertebrates 2. Bucks, Commonwealth Agricultural Bureaux, Farnham Royal, 27p.

KoHn, A. \& B.M.M. FernANDES. 1987. Estudo comparativo dos helmintos parasitos de peixes do rio Mogi Guassu, coletados nas excursões realizadas entre 1927 e 1985. Mem. Inst. Oswaldo Cruz 82 (4): 483-500.

. 1988a. Helminth parasites of fishes from the hydroeletric power station of Eletrosul (Brazil). I-Procamallamus petterae n.sp. and Spirocamallanus pintoi n.sp. (Nematoda, Camallanidea) from the reservoir of "Salto Osório". Mem. Inst. Oswaldo Cruz 83 (3): 293-298.

_. 1988b. Procamallanus annipetterae nom. nov. for Procamallanus petterae Kohn \& Fernandes, 1988 preocupied by Procamallanus (Procamallanus) petterae Moravec \& Sey, 1988. Mem. Inst. Oswaldo Cruz 83: 535.

KoHn, A.; B.M.M. FernANDES; H.V. PIPOLO \& M.P. Godoy. 1988. Helmintos parasitas de peixes das usinas hidrelétricas da Eletrosul (Brasil). II. Reservatórios de Salto Osório e 
de Salto Santiago, Bacia do Rio Iguaçu. Mem. Inst. Oswaldo Cruz 83: 299-303.

Moravec, F. \& O. Sey. 1988. Nematodes of freshwater fishes Part. 1. Camallanoidea and Habronematoidea. Vest. Cesk. Spolec. Zool. 52: 128-148.

Moravec, F. \& V. Thatcher. 1997a. New data on the morphology and systematic status of Klosinemella iheringi (Nematoda: Atractidae) from an Amazonian serrasalmid fish. Folia Parasitol. 44: 48-54.

$.1997 \mathrm{~b}$. Raphidascaroides brasiliensis n. sp (Nematoda: Anisakidae), an intestinal parasite of the thorny catfish Pterodoras granulosus from Amazonia, Brazil. Syst. Parasitol. 38: 65-71.

Moravec, F.; A. KoHn \& B.M.M. Fernandes. 1990. First record of Raphidascaris (Sprentascaris) hypostomi (Petter et Cassone, 1984) comb.n. and R. (S.) mahnerti (Petter et Cassone, 1984) comb.n. (Nematoda: Anisakidae) from Brazil with remarks on the taxonomic status of the genus Sprentascaris Petter et Cassone, 1984. Folia Parasitol. 37: 131-140.

-1992a. Three new species of oxyuroid nematodes, including two new genera, from freshwater catfishes in Brazil. Syst. Parasitol. 21: 189-201.

- 1992b. Nematode parasites of fishes of the Paraná River, Brazil. Part 1. Trichuroidea, Oxyuroidea and Cosmocercoidea. Folia Parasitol. 39: 327-353.

1992c. Neoparaseuratum travassosi n.g., n.sp. (Nematoda: Quimperiidae), a new parasite from the thorny catfish Pterodoras gramulosus in Brazil. Mem. Inst. Oswaldo Cruz 87 (Suppl. I): 145-150.

1993a. Nematode parasites of fishes of the Paraná River, Brazil. Part 2. Seuratoidea, Ascaridoidea, Habronematoidea and Acuarioidea. Folia Parasitol. 40: 115-134.

. 1993b. Nematode parasites of fishes of the Paraná River, Brazil. Part 3. Camallanoidea and Dracunculoidea. Folia Parasitol. 40: 211-229.

1993c. Travassosnema travassosi paranaensis subsp.n. and first description of the female of Guyanema raphiodoni Moravec, Kohn and Fernandes, 1993 (Nematoda: Guyanemidae), dracunculoid parasites of characid fishes in Brazil. Ann. Parasitol. Hum. Comp. 68 (5/6): 229-233.

1994a. Two new species of the genus Goezia, G. brasiliensis sp.n. and G. brevicaeca sp.n. (Nematoda: Anisakidae), from freshwater fishes in Brazil. Folia Parasitol. 41: 271-278.

1994b. Structure of the cephalic end of two little-known oxyuroid genera, Travnema Pereira, 1938 and Cosmoxynemoides Travassos, 1949, parasites of fishes, as revealed by SEM. Jour. Helminthol. 68: 319-322.

1997. New observations on seuratoid nematodes parasitic in fishes of the Paraná River, Brasil. Folia Parasitol. 44: 209-223.

Moreira, N.I.B.; C.L. Oliveira \& H.M.A. CostA. 1991. A new helminth parasite of fish: Spirocamallanus freitasi sp.n. (Nematoda - Camallanidae). Mem. Inst. Oswaldo Cruz 86: 429-431.

- 1994. Spirocamallanus inopinatus (Travassos, Artigas \& Pereira, 1928) e Spirocamallanus saofranciscencis sp.n. (Nematoda, Camallanidae) em peixes da Represa de Tres Marias. Arq. Bras. Med. Vet. Zoot. 46 (5): 485-500.

PEREIRA, JR. J. \& M.A.S. CosTA. 1996. Cucullanidae (Nematoda: Seuratoidea) em Micropogonias furnieri (Desmarest, 1823) (Sciaenidae) do Rio Grande do Sul, com a descrição de Cucullanus cassinensis sp.n. e Dichelyne (Dichelyne) micropogonii. Com. Mus. Cienc. Tecnol. PUCRS, Ser. Zool., Porto Alegre, 9: 11-30.

PETTER, A.J. 1974a. Deux nouvelles especes de Nématodes Camallanina parasites de Hoplerythrinus unitaeniatus (Characidae, Cypriniformes) em Guyane; création d'une nouvelle famille: les Guyanemidae (Dracunculoidea) Bull. Mus. natn. Hist. nat., Paris 
$3^{\text {e }}$ sér no. 232. Zool., 156: 803-812.

. 1974b. Essai de classification de la famille des Cucullanidae. Bull. Mus. natn.

Hist. nat., $3^{\mathrm{e}}$ sér 255. Zool., 177: 1469-1490.

. 1979. Essai de classification de la sous-famille Camallaninae (Nematoda, Camallanidae). Bull. Mus. natn. Hist. nat., Paris 4 e sér, 1 (4): 991-1008.

1990. Nématodes de poissons du Paraguay. VI Description de deux nouvelles espéces du genre Spirocamallamus et compleménts à la description de Procamallanus annipetterae Kohn \& Fernandes, 1988. Rev. Suisse Zool 97 (2): 327-338.

PETTER, A.J. \& J.C. QUENTIN. 1976. Key to the genera of Oxyuroidea. In: R.C. ANDERSON, R.C.; A.G. ChaBAUD \& S. WiLlmotT (Eds). CIH Keys to the nematode parasites of vertebrates 4. Bucks, Commonwealth Agricultural Bureaux, Farnham Royal, 30p.

PETTER, A.J. \& V.E. THATCHER. 1988. Observations sur la structure de la capsule buccale de Spirocamallanus inopinatus (Nematoda) parasite de Poissons brésiliens. Bull. Mus. natn. Hist. nat., Paris 4 é sér., 10: 685-692.

PINTO, R.M.; J.J. VICENTE; D. NORONHA \& S.P. FÁBIO. 1988. Redescription of Oncophora melanocephala (Rudolphi, 1819) Baudin-Laurencin, 1971 (Nematoda, Camallanidae). Mem. Inst. Oswaldo Cruz 83 (2): 233-237.

PinTO, R.M.; J.J. VicENTE \& D. NoRONHA. 1992. On some family related parasites (Nematoda; Cucullanidae) from the marine fish Paralonchurus brasiliensis (Steindachner, 1875) (Pisches, Ostraciidae). Mem. Inst. Oswaldo Cruz 87 (Suppl. I): 207-212.

RÊGo, A.A.; J. Carvajal \& G. SchaEFFER. 1985. Patogenia del higado de peces (Pagrus pagrus L.) provocada por larvas de nematodos Anisakidae. Parasitol. al Dia 9: 75-79,

RÊGO, A.A. \& J.C. EIRAS. 1988. Ecologia da parasitose de peixes e aves do rio Cuiabá (Mato Grosso, Brasil) por Eustrongylides ignotus (Nematoda, Dioctophymidae). Actas Col. Luso-Esp. Ecol. Bacias Hidrogr. e Rec. Zoológicos: 335-341,

RÊGO, A.A. \& J.J. VICENTE. 1988. Eustrongylides ignotus Jägerskiöld, 1909 (Nematoda: Dioctophymatoidea), parasito de peixes, anfibios, répteis e aves. Distribuição e taxonomia. Ciênc. Cult. 40 (5): 478-483.

Rodrigues, H.O.; R.M. PINTO \& D. NoronHa. 1991. The species of Brazilian Procamallanus with general considerations (Nematoda, Camallanoidea). Mem. Inst. Oswaldo Cruz 86 (1): 107-113.

SARMENTO, T.M.; E. ForTes \& R.P. HofFmanN. 1995. Cucullanus debacoi sp.n. Nematoda, parasito do intestino de Pimelodus maculatus Lacépède, 1803 (Pisces, Pimelodidae) do rio Guaiba, Porto Alegre, Rio Grande do Sul. A Hora Veterinária 15 (85): 38-40.

THATCHER, V.E. 1991. Amazon fish parasites. Amazoniana 11 (3/4): 263-570.

VICENTE, J.J.; R.M. PinTo \& O. AGUILERA. 1989. On Dichelyne (Cucullanellus) elongatus (Tornquist, 1931) Petter, 1974: South American correlated species (Nematoda, Cucullanidae) and some other helminths of Micropogonias furnieri (Desmarest, 1823) (Pisces, Sciaenidae). Mem. Inst. Oswaldo Cruz 84 (3): 357-361.

ViCEnTE, J.J.; H.O. Rodrigues \& D.C. Gomes. 1985. Nematóides do Brasil $1^{\text {a }}$ parte: Nematóides de peixes. Atas Soc. Biol. Rio de Janeiro 25: 1-79.

Recebido em 02.IV.1998; aceito em 19.VII.1999 
Tabela I. Brasilnema pimelodellae; Parasynodontisia petterae; Ichthyouris brasiliensis; Ichthyouris laterifilamenta; Travnema araujoi. Medidas em milimetros, segundo MORAVEC et al. 1992.

\begin{tabular}{|c|c|c|c|c|c|c|c|c|c|c|}
\hline & \multicolumn{2}{|c|}{ B. pimelodellae } & \multicolumn{2}{|c|}{ P. petterae } & \multicolumn{2}{|c|}{ 1. brasiliensis } & \multicolumn{2}{|c|}{ 1. laterifilamenta } & \multicolumn{2}{|c|}{ T. araujoi } \\
\hline & Macho & Fêmea & Macho & Fêmea & Macho & Fêmea & Macho & Fêmea & Macho & Fémea \\
\hline Comprimento & $0,990-1,200$ & $1,960-2,640$ & $1,390-2,460$ & $2,350-4,090$ & $1,280-1,880$ & $3,160-4,500$ & $1,160-1,440$ & $2,560-3,330$ & 2,350 & $4,620-5,780$ \\
\hline Largura & $0,082-0,136$ & $0,249-0,326$ & $0,082-0,122$ & $0,177-0,340$ & $0,190-0,150$ & $0,231-0,422$ & $0,082-0,109$ & 0,190-0,299 & 0,204 & $0,367-0,490$ \\
\hline Cápsula bucal & $\begin{array}{c}0,003-0,005 \\
\times \\
0,008-0,009\end{array}$ & $\begin{array}{c}0,006-0,009 \\
\times \\
0,018-0,021\end{array}$ & - & - & $\begin{array}{c}0,012-0,015 \\
x \\
0,018\end{array}$ & $\begin{array}{c}0,021-0,030 \\
\times \\
0,021-0,024\end{array}$ & - & - & $\begin{array}{l}0,015 \\
\times \\
0,027\end{array}$ & $\begin{array}{c}0,015-0,18 \\
\times \\
0,042-0,048\end{array}$ \\
\hline Esôfago & $0,195-0,222$ & $0,300-0,348$ & $0,597-0,702$ & $0,80-1,22$ & $0,294-0,333$ & $0,516-0,585$ & $0,231-0,264$ & $0,444-0,501$ & 0.249 & $0,345-0,396$ \\
\hline Faringe & $\begin{array}{c}0,004-0,006 \\
x \\
0,015-0,018\end{array}$ & $\begin{array}{c}0,006-0,008 \\
x \\
0,024-0,036\end{array}$ & $\begin{array}{c}0,006-0,009 \\
x \\
0,015-0,021\end{array}$ & $\begin{array}{c}0,018 \\
x \\
0,033-0,048\end{array}$ & $\begin{array}{c}0,213-0,243 \\
x \\
0,024-0,030\end{array}$ & $\begin{array}{c}0,387-0,435 \\
x \\
0,060-0,078\end{array}$ & $\begin{array}{c}0,165-0,186 \\
x \\
0,018-0,024\end{array}$ & $\begin{array}{c}0,345-0,387 \\
x \\
0,039-0,048\end{array}$ & $\begin{array}{c}0,093 \\
x \\
0,060\end{array}$ & $\begin{array}{c}0,126-0,141 \\
x \\
0,096-0,102\end{array}$ \\
\hline Istmo & $\begin{array}{c}0,033-0,036 \\
x \\
0,015-0,021\end{array}$ & $\begin{array}{c}0,024-0,030 \\
x \\
0,030-0,036\end{array}$ & $\begin{array}{c}0,018-0,030 \\
x \\
0,015-0,018\end{array}$ & $\begin{array}{c}0,018-0,030 \\
\times \\
0,027-0,045\end{array}$ & $\begin{array}{c}0,015-0,018 \\
x \\
0,018-0,021\end{array}$ & $\begin{array}{c}0,006-0,009 \\
\times \\
0,012-0,036\end{array}$ & $\begin{array}{c}0,012-0,015 \\
x \\
0,015\end{array}$ & $\begin{array}{c}0,009-0,012 \\
\times \\
0,027-0,033\end{array}$ & $\begin{array}{l}0,081 \\
\times \\
0,048\end{array}$ & $\begin{array}{c}0,081-0,108 \\
x \\
0,063-0,066\end{array}$ \\
\hline Bulbo & $\begin{array}{c}0,048-0,057 \\
x \\
0,042-0,051\end{array}$ & $\begin{array}{c}0,078-0,099 \\
\times \\
0,075-0,099\end{array}$ & $\begin{array}{c}0,063-0,078 \\
x \\
0,015-0,021\end{array}$ & $\begin{array}{c}0,096-0,147 \\
\times \\
0,078-0,126\end{array}$ & $\begin{array}{c}0,063-0,072 \\
x \\
0,063-0,075\end{array}$ & $\begin{array}{c}0,120-0,135 \\
\times \\
0,126-0,159\end{array}$ & $\begin{array}{c}0,054-0,063 \\
\times \\
0,048-0,057\end{array}$ & $\begin{array}{c}0,087-0,0105 \\
\times \\
0,090-0,114\end{array}$ & $\begin{array}{c}0,075 \\
\times \\
0,075\end{array}$ & $\begin{array}{c}0,129-0,159 \\
x \\
0,111-0,135\end{array}$ \\
\hline Anel nervoso & $0,111-0,129$ & $0,153-0,177$ & $0,135-0,231$ & $0,204-0,258$ & $0,135-0,162$ & $0,165-0,198$ & $0,111-0,135$ & $0,159-0,168$ & 0,147 & $0,183-0,204$ \\
\hline Poro excretor & $0,264-0,294$ & $0,462-0,653$ & $0,680-0,966$ & $0,680-0,966$ & $0,354-0,571$ & $0,625-0,993$ & $0,375-0,456$ & $0,625-0,707$ & 0,666 & $1,060-1,400$ \\
\hline Espiculo & $0,045-0,057$ & - & $0,042-0,063$ & - & $0,060-0,069$ & - & $0,036-0,045$ & - & 0,063 & - \\
\hline Cone genital & - & - & $0,009-0,018$ & - & - & - & - & - & 0,015 & - \\
\hline Cauda & $0,0245-0,326$ & - & $0,120-0,270$ & $0,153-0,721$ & $0,099-0,138$ & $0,639-0,870$ & $0,063-0,087$ & $0,480-0,600$ & 0,219 & $0,201-0,270$ \\
\hline Vulva & - & $0,666-0,843$ & - & $1,24-1,90$ & - & $1,130-1,650$ & - & $0,857-0,938$ & - & $2,050-2,760$ \\
\hline $\begin{array}{c}\text { Ovos comprimento } \\
x \\
\text { largura }\end{array}$ & - & $\begin{array}{c}0,144-0,156 \\
x \\
0,063-0,096\end{array}$ & - & $\begin{array}{c}0,285-0,422 \\
x \\
0,081-0,099\end{array}$ & - & $\begin{array}{c}0,093-0,099 \\
x \\
0,033-0,036\end{array}$ & - & $\begin{array}{c}0,117-0,138 \\
x \\
0,051-0,069\end{array}$ & - & $\begin{array}{c}0,225-0,255 \\
x \\
0,069-0,090\end{array}$ \\
\hline Asas caudais & - & - & - & - & $0,039-0,045$ & - & $0,012-0,015$ & - & - & - \\
\hline Espinho caudal & - & $0,517-0,666$ & - & - & $0,063-0,087$ & $0,612-0,734$ & - & $0,414-0,495$ & - & - \\
\hline
\end{tabular}




\begin{tabular}{|c|c|c|c|c|c|c|c|c|}
\hline & \multirow{2}{*}{$\begin{array}{c}\text { C. brevispiculus } \\
\text { Macho }\end{array}$} & \multicolumn{2}{|c|}{ C. grandistomis } & \multicolumn{2}{|c|}{ C. patoi } & \multirow{2}{*}{$\begin{array}{c}\text { C. pimelodellae } \\
\text { Macho }\end{array}$} & \multicolumn{2}{|c|}{ C. pseudoplatistomae } \\
\hline & & Macho & Fêmea & Macho & Fềmea & & Macho & Fêmea \\
\hline Comprimento & 1,360 & $3,700-4,700$ & $3,000-7,040$ & $8,970-9,480$ & 4,280 & $3,740-4,920$ & $9,090-11,830$ & $8,570-15,160$ \\
\hline Largura & 0,313 & $0,090-0,160$ & $0,066-0,150$ & 0,340 & 0,200 & $0,163-0,190$ & $0,177-0,272$ & $0,204-0,272$ \\
\hline Esôfago & 0,830 & $0,560-0,680$ & $0,450-0,790$ & $0,950-0,980$ & 0,350 & $0,577-0,666$ & $1,090-1,440$ & $1,100-1,470$ \\
\hline Anel nervoso & 0,313 & $0,210-0,270$ & $0,240-0,300$ & $0,350-0,480$ & 0,320 & $0,204-0,207$ & $0,313-0,408$ & $0,326-0,422$ \\
\hline Poro excretor & 0,734 & $0,310-0,370$ & $0,260-0,310$ & - & - & $0,326-0,353$ & $0,639-0,775$ & $0,520-0,762$ \\
\hline Ventosa pré-anal & - & - & - & $0,150-0,170$ & - & - & - & - \\
\hline Espiculos & 0,225 & 0,780 & - & $0,470-0,560$ & - & $0,210-0,330$ & $0,486-0,534$ & - \\
\hline Gubernáculo & 0,090 & - & - & 0,060 & - & $0,036-0,045$ & $0,775-0,084$ & - \\
\hline Vulva & - & - & $2,200-5,100$ & - & - & - & - & $5,980-8,770$ \\
\hline $\begin{array}{c}\text { Ovos comprimento } \\
x \\
\text { largura }\end{array}$ & - & - & - & - & - & - & - & $\begin{array}{c}0,045-0,060 \\
x \\
0,030-0,045\end{array}$ \\
\hline Reto & - & - & - & - & 0,070 & - & - & - \\
\hline Ventosa-cloaca & - & - & - & $0,500-0,600$ & - & - & - & - \\
\hline Cloaca & - & $0,102-0,140$ & - & $0,210-0,240$ & - & - & - & - \\
\hline Ånus & - & - & $0,100-0,150$ & - & 0,190 & - & - & - \\
\hline Deiridios & 0,721 & 0,400 & $0,360-0,480$ & - & - & - & $0,639-0,721$ & $0,544-0,721$ \\
\hline Cauda & 0,218 & - & - & - & - & - & $0,150-0,198$ & $0,290-0,394$ \\
\hline
\end{tabular}


Tabela III. Cucullanus riograndensis (segundo FORTES et al. 1993); Dichelyne (C.) elongatus (segundo PINTO et al. 1992); Dichelyne leporini (segundo MORAVEC et al. 1993); Neoparaseuratum travassosi (segundo MoRAVEC et al. 1992). Medidas em milimetros.

\begin{tabular}{|c|c|c|c|c|c|c|c|c|}
\hline & \multicolumn{2}{|c|}{ C. riograndensis } & \multicolumn{2}{|c|}{ D. (C.) elongatus } & \multicolumn{2}{|c|}{ D. leporini } & \multicolumn{2}{|c|}{ N. travassoi } \\
\hline & Macho & Fêmea & Macho & Fêmea & Macho & Fêmea & Macho & Fêmea \\
\hline Comprimento & 7,350 & 8,430 & 4,900 & 8,200 & 9,720 & $17,710-23,87$ & $3,140-6,740$ & 11,400 \\
\hline Largura & 0,190 & 0,230 & 0,350 & 0,420 & 0,218 & $0,462-0,707$ & $0,122-0,231$ & 0,381 \\
\hline Esôfago & 0,740 & 0,750 & 0,700 & 0,830 & 1,140 & $1,410-1,650$ & $0,449-0,680$ & 0,843 \\
\hline Anel nervoso & - & 0,290 & 0,250 & 0,280 & 0,272 & $0,340-0,367$ & $0,210-0,318$ & 0,354 \\
\hline Poro excretor & - & - & 0,430 & 0,640 & - & $1,050-1,280$ & $0,513-0,938$ & 1,200 \\
\hline Ventosa pré-anal & - & - & 0,240 & - & - & - & - & - \\
\hline Espiculos & 0,530 & - & 0,630 & - & 0,660 & - & $0,159-0,303$ & - \\
\hline Gubernáculo & 0,360 & - & 0,070 & - & 0,090 & - & $0,060-0,075$ & - \\
\hline Vulva & - & - & - & 4,80 & - & $12,880-15,010$ & - & 7,240 \\
\hline Ovejetor & - & - & - & 0,280 & - & - & - & - \\
\hline $\begin{array}{c}\text { Ovos comprimento } \\
x \\
\text { largura }\end{array}$ & - & - & - & 0,100 & - & $\begin{array}{c}0,060-0,081 \\
x \\
0,051-0,060\end{array}$ & - & $\begin{array}{c}0,090-0,105 \\
x \\
0,060-0,066\end{array}$ \\
\hline Reto & - & - & 0,500 & 0,500 & $\begin{array}{l}0,925 \\
x \\
0,082\end{array}$ & $\begin{array}{c}1,200-1,270 \\
x \\
0,095-0,136\end{array}$ & - & - \\
\hline Ceco intestinal & - & - & 0,120 & - & 0,218 & - & $0,111-0,129$ & - \\
\hline Cloaca & - & - & - & 0,180 & - & $0,367-0,422$ & - & 0,141 \\
\hline Deiridios & - & - & - & - & & $1,160-1,350$ & $0,378-0,546$ & 0,694 \\
\hline
\end{tabular}


Tabela IV. Raphidascaris (S.) hypostomi (segundo MORAVEC et al. 1990); Raphidascaris (S.) mahnerti (segundo MoRAVEC et al. 1990); Camallanus acaudatus (segundo FERRAZ \& THATCHER 1990); Camallanus tridentatus (segundo FERRAZ \& THATCHER 1990); Oncophora melanocephala (segundo PINTO et al. 1988). Medidas em milímetros.

\begin{tabular}{|c|c|c|c|c|c|c|c|c|c|c|}
\hline & \multicolumn{2}{|c|}{ R. (S.) hypostomi } & \multicolumn{2}{|c|}{ R. (S.) mahnerti } & \multicolumn{2}{|c|}{ C. acaudatus } & \multicolumn{2}{|c|}{ C. tridentatus } & \multicolumn{2}{|c|}{ O. melanocephala } \\
\hline & Macho & Fêmea & Macho & Fêmea & Macho & Fêmea & Macho & Fêmea & Macho & Fêmea \\
\hline Comprimento & $6,060-7,900$ & $11,220-14,270$ & $5,280-6,050$ & $6,030-8,980$ & $1,000-1,800$ & $12,000-19,000$ & 3,600 & $10,000-12,000$ & 14,620 & 23,290 \\
\hline Largura & $0,340-0,829$ & $0,802-1,140$ & $0,150-0,218$ & $0,231-0,313$ & $0,080-0,130$ & $0,320-0,440$ & 0,100 & $0,170-0,230$ & $0,230-0,300$ & 0,280 \\
\hline Cápsula bucal & - & - & - & - & $\begin{array}{c}0,050-0,065 \\
x \\
0,057-0,107\end{array}$ & $\begin{array}{c}0,067-0,095 \\
x \\
0,075-0,107\end{array}$ & $\begin{array}{c}0,062 \\
x \\
0,075\end{array}$ & $\begin{array}{c}0,110-0,120 \\
x \\
0,125-0,170\end{array}$ & $\begin{array}{c}0,120-0,140 \\
x \\
0,140-0,160\end{array}$ & $\begin{array}{c}0,013 \\
x \\
0,015\end{array}$ \\
\hline Anel quitinoso & - & - & - & - & $\begin{array}{c}0,010-0,015 \\
x \\
0,062-0,070\end{array}$ & $\begin{array}{c}0,010-0,020 \\
x \\
0,075-0,095\end{array}$ & $0,015-0,042$ & $\begin{array}{c}0,015-0,022 \\
x \\
0,067-0,075\end{array}$ & $\begin{array}{c}0,056 \\
x \\
0,091\end{array}$ & $\begin{array}{c}0,043 \\
x \\
0,093\end{array}$ \\
\hline Esôfago muscular & $0,800-1,330$ & $1,220-1,390$ & $0,720-0,855$ & $0,880-1,140$ & $0,310-0,490$ & $0,360-0,500$ & $0,400-0,042$ & $0,700-0,825$ & $1,340-1,540$ & 1,610 \\
\hline Esôfago glandular & - & - & - & - & $0,355-0,397$ & $0,630-0,750$ & - & - & $1,190-1,400$ & 1,470 \\
\hline Apéndice ventricular & $0,150-0,204$ & $0,190-0,204$ & $0,072-0,078$ & $0,063-0,120$ & - & - & - & - & - & - \\
\hline Anel nervoso & $0,272-0,435$ & $0,408-0,503$ & $0,246-0,321$ & $0,249-0,399$ & $0,112-0,177$ & 0,200 & 0.170 & $0,250-0,300$ & $0,290-0,330$ & 0,430 \\
\hline Poro excretor & $0,354-0,462$ & $0,462-0,490$ & $0,267-0,339$ & $0,279-0,411$ & $0,310-0,347$ & $0,500-0,700$ & - & - & $1,040-1,300$ & 1,630 \\
\hline Espiculo & - & - & - & - & $0,155-0,197$ & - & 0,205 & - & - & - \\
\hline Espiculos & $0,273-0,294$ & - & $0,123-0,129$ & - & - & - & - & - & $0,700-0,770$ & 0,540 \\
\hline Cloaca/ânius & - & - & - & - & $0,047-0,057$ & - & 0,063 & - & $0,100-0,140$ & 0,290 \\
\hline Tridentes & - & - & - & - & - & - & - & - & $0,210-0,26$ & 0,250 \\
\hline Vulva & - & $1,860-2,310$ & - & $1,820-3,030$ & - & $8,000-12,000$ & - & $5,000-7,000$ & - & 6,020 \\
\hline $\begin{array}{c}\text { Ovos comprimento } \\
x \\
\text { largura }\end{array}$ & - & $\begin{array}{c}0,054-0,066 \\
\times \\
0,048-0,057\end{array}$ & - & $\begin{array}{c}0,078-0,087 \\
x \\
0,063-0,075\end{array}$ & - & - & - & - & - & - \\
\hline Ventriculo & $\begin{array}{c}0,081-0,136 \\
x \\
0,218-0,326\end{array}$ & $\begin{array}{c}0,122-0,136 \\
x \\
0,299-0,408\end{array}$ & $\begin{array}{c}0,033-0,060 \\
\times \\
0,093-0,096\end{array}$ & $\begin{array}{c}0,060-0,063 \\
x \\
0,096-0,123\end{array}$ & - & - & - & - & - & - \\
\hline Asa caudal & - & - & $0,012-0,021$ & - & $\begin{array}{c}0,147-0,267 \\
x \\
0,012-0,017\end{array}$ & - & $0,292-0,022$ & - & - & - \\
\hline
\end{tabular}


Tabela V. Paracamallanus amazonensis (segundo MoRAVEC et al. 1993); Procamallanus (P.) annipetterae (segundo KoHN \& FERnANdes 1988); Procamallanus (S.) freitasi (segundo MoReIRA et al. 1991); Procamallanus (S.) pintoi (segundo KoHN \& FERNANDES 1988); Guyanema raphidioni (segundo MORAVEC et al. 1993). Medidas em milimetros.

\begin{tabular}{|c|c|c|c|c|c|c|c|c|c|c|}
\hline & \multicolumn{2}{|c|}{ P. amazonensis } & \multicolumn{2}{|c|}{ P. (P.) annipetterae } & \multicolumn{2}{|c|}{ P. (S.) freitasi } & \multicolumn{2}{|c|}{ P. (S.) pintoi } & \multicolumn{2}{|c|}{ G. raphidioni } \\
\hline & Macho & Fêmea & Macho & Fêmea & Macho & Fêmea & Macho & Fêmea & Macho & Fêmea \\
\hline Comprimento & $3,050-4,390$ & $4,870-6,490$ & 9,690 & 21,800 & $4,360-7,400$ & $11,300-12,980$ & 4,670 & $21,800-26,000$ & 3,700 & 4,240 \\
\hline Largura & $0,082-0,122$ & 0,150 & 0,500 & $\begin{array}{c}0,180 \\
x \\
0,187\end{array}$ & $0,121-0,150$ & $0,227-0,266$ & 0,250 & $0,950-1,160$ & 0,068 & 0,069 \\
\hline Cápsula bucal & $0,081-0,087$ & $0,096-0,099$ & $\begin{array}{c}0,131 \\
x \\
0,123\end{array}$ & 0,644 & $\begin{array}{c}0,047 \\
x \\
0,021-0,027\end{array}$ & $\begin{array}{c}0,047-0,050 \\
x \\
0,021-0,024\end{array}$ & - & $\begin{array}{c}0,060-0,071 \\
x \\
0,049-0,056\end{array}$ & - & - \\
\hline Tridente & $0,069-0,075$ & $0,075-0,081$ & - & - & - & - & - & - & - & - \\
\hline Esôfago muscular & $0,462-0,639$ & $0,653-0,666$ & 0,625 & 0,887 & $0,369-0,412$ & $0,412-0,492$ & 0,288 & $0,266-0,296$ & 0,245 & 0,195 \\
\hline Esofago glandular & $0,340-0,517$ & $0,544-0,612$ & 0,868 & - & $0,455-0,623$ & $0,622-0,811$ & 0,587 & $0,862-1,045$ & 1,220 & 1,100 \\
\hline Espiculos & - & - & - & - & - & - & - & - & 0,150 & - \\
\hline Espiculo maior & $0,171-0,195$ & - & 0,021 & - & $0,465-0,534$ & - & 0,094 & - & - & - \\
\hline Espiculo menor & $0,036-0,057$ & - & 0,016 & - & $0,172-0,214$ & - & 0,082 & - & - & - \\
\hline Anel nervoso & $0,163-0,190$ & $0,177-0,190$ & 0,298 & - & $0,184-0,219$ & $0,219-0,239$ & 0,112 & $0,120-0,158$ & 0,129 & 0,129 \\
\hline Poro excretor & $0,272-0,345$ & $0,354-0,381$ & - & - & $0,270-0,300$ & $0,291-0,339$ & 0,156 & 0,300 & 0,141 & - \\
\hline Vulva & - & $1,970-2,530$ & - & - & - & $4,010-4,800$ & - & 7,900 & - & - \\
\hline Cloaca & - & - & 0,336 & - & - & - & 0,120 & - & 0,102 & 0,126 \\
\hline Ânus & - & $0,126-0,135$ & - & 0,281 & - & - & - & $0,223-0,392$ & - & - \\
\hline Reto & - & - & - & 0,373 & - & - & - & 0,279 & - & - \\
\hline Deiridios & - & - & - & - & $0,162-0,196$ & $0,206-9,236$ & - & - & - & 0,789 \\
\hline
\end{tabular}


Tabela VI. Travassosnema travassosi (segundo COSTA et al. 1991); Travassosnema travassosi paranaensis (segundo MoRAVEC et al. 1993); Goezia brasiliensis; Goezia brevicaeca (segundo MORAVEC et al. 1994). Medidas em milimetros.

\begin{tabular}{|c|c|c|c|c|c|c|c|c|}
\hline & \multicolumn{2}{|c|}{ T. travassoi } & \multicolumn{2}{|c|}{ T. t. paranaensis } & \multicolumn{2}{|c|}{ G. brasiliensis } & \multicolumn{2}{|c|}{ G. brevicaeca } \\
\hline & Macho & Fêmea & Macho & Fêmea & Macho & Fèmea & Macho & Fêmea \\
\hline Comprimento & $2,040-2,440$ & $7,530-13,820$ & 1,610 & 2,920 & 11,140 & $9,720-15,670$ & 17,150 & 22,580 \\
\hline Largura & $0,033-0,049$ & $0,136-0,244$ & 0,041 & 0,136 & 1,120 & $0,789-1,006$ & 0,707 & 0,938 \\
\hline Esôfago & - & - & - & - & 0,041 & $0,041-0,068$ & 0,054 & 0,054 \\
\hline Esôfago muscular & $0,086-0,140$ & - & 0,108 & 0,135 & - & - & - & - \\
\hline Esôfago glandular & $0,146-0,160$ & - & 0,141 & 0,144 & - & - & - & - \\
\hline Ventriculo & - & - & - & - & $\begin{array}{c}0,095 \\
x \\
0,136\end{array}$ & $\begin{array}{c}0,082-0,095 \\
x \\
0,095-0,150\end{array}$ & $\begin{array}{c}0,095 \\
x \\
0,150\end{array}$ & $\begin{array}{c}0,122 \\
x \\
0,163\end{array}$ \\
\hline Ceco intestinal & - & - & - & - & 0,354 & $0,326-0,544$ & 0,109 & 0,109 \\
\hline Ceco esofagiano & - & - & $\begin{array}{c}0,366 \\
x \\
0,033\end{array}$ & $\begin{array}{c}0,618 \\
x \\
0,084\end{array}$ & 1,92 & $2,630-2,770$ & 3,010 & 0,48 \\
\hline Apéndice esofagiano & $0,532-0,785$ & $1,002-1,662$ & - & - & - & - & - & - \\
\hline Espiculos & $0,050-0,060$ & - & 0,045 & - & 0,802 & - & 0,367 & - \\
\hline Anel nervoso & - & $0,106-0,181$ & 0,066 & 0,099 & 0,299 & $0,299-0,340$ & 0,299 & 0,354 \\
\hline Poro excretor & - & - & 0,090 & 0,105 & - & - & - & - \\
\hline Vulva & - & $0,329-0,612$ & - & 0,363 & - & $5,110-6,280$ & - & 9,520 \\
\hline Cloaca & - & - & - & - & 0,095 & - & 0,082 & - \\
\hline Ánus & - & - & - & - & - & $0,272-0,313$ & - & 0,204 \\
\hline Ovejetor & - & - & - & $0,273-0,306$ & - & - & - & - \\
\hline Ovos & - & - & - & - & - & $\begin{array}{l}0,042 \\
x \\
0,045\end{array}$ & - & 0,042 \\
\hline Gubernáculo & $0,013-0,018$ & - & 0,009 & - & - & - & - & - \\
\hline Processos digitiformes da cauda & - & - & - & - & 0,027 & $0,068-0,082$ & 0,025 & - \\
\hline
\end{tabular}


Tabela VII. Spectatus sp. (segundo MORAVEC et al. 1997); Cucullanus pinnai pterodorasi (segundo MoRAVEC et al. 1997); Cucullanus rhamphichthydis (segundo MORAVEC et al. 1997); Dichelyne pimelodi (segundo MoRAVEC et al. 1997); Seuratoidea sp. (segundo MORAVEC et al. 1997). Medidas em milímetros.

\begin{tabular}{|c|c|c|c|c|c|c|}
\hline & \multirow{2}{*}{$\begin{array}{c}\text { Spectatus sp. } \\
\text { Fêmea }\end{array}$} & \multicolumn{2}{|c|}{ C. pinnai pterodorasi } & \multirow{2}{*}{$\begin{array}{c}\text { C. rhamphichthydis } \\
\text { Fêmea }\end{array}$} & \multirow{2}{*}{$\frac{\text { D. pimelodi }}{\text { Macho }}$} & \multirow[t]{2}{*}{ Seuratoidea sp. } \\
\hline & & Macho & Fêmea & & & \\
\hline Comprimento & 11,700 & $6,830-9,900$ & $8,600-10,350$ & $9,340-12,350$ & 6,900 & $5,940-6,170$ \\
\hline Largura & 0,571 & $0,272-0,354$ & $0,313-0,394$ & $0,204-0,218$ & 0,435 & 0,204 \\
\hline Esőfago total & 1,670 & $0,748-0,884$ & $0,843-0,870$ & $1,170-1,350$ & 0,911 & $0,571-0,585$ \\
\hline Oesophastome & - & $\begin{array}{c}0,272-0,313 \\
x \\
0,150-0,177\end{array}$ & $0,299-0,177$ & $\begin{array}{c}0,258 \\
x \\
0,190\end{array}$ & $\begin{array}{l}0,218 \\
x \\
0,136\end{array}$ & - \\
\hline Anel nervoso & 0,408 & $0,313-0,408$ & $0,381-0,408$ & $0,354-0,476$ & 0,340 & $0,126-0,189$ \\
\hline Poro excretor & 1,250 & $0,585-0,789$ & $0,625-0,680$ & $1,810-2,30$ & 1,140 & $0,198-0,225$ \\
\hline Espiculos & - & $0,544-0,558$ & - & - & 1,460 & - \\
\hline Gubernáculo & - & 0,069 & - & - & 0,128 & - \\
\hline Cauda & 0,422 & $0,231-0,245$ & $0,286-0,299$ & $0,313-0,326$ & 0,231 & $0,163-0,190$ \\
\hline Deirídios & - & $0,544-0,748$ & $0,558-0,680$ & 1,630 & $0,979-1,020$ & $0,653-0,666$ \\
\hline Vulva & 3,940 & - & $3,540-5,070$ & $2,870-4,150$ & - & - \\
\hline Ovos & - & - & $\begin{array}{c}0,0480 \\
x \\
0,033\end{array}$ & - & - & - \\
\hline Faringe & $\begin{array}{c}0,054 \\
x \\
0,082\end{array}$ & - & - & - & - & - \\
\hline Esôfago muscular & 1,130 & - & - & - & - & - \\
\hline Esōfago glandular & 0,490 & - & - & - & - & - \\
\hline Ceco intestinal & - & - & - & - & $\begin{array}{l}0,286 \\
x \\
0,082\end{array}$ & - \\
\hline Primórdio genital & - & - & - & - & - & 3,550 \\
\hline
\end{tabular}


Tabela VIII. Dichelyne (C.) amaruincai; Dichelyne (C.) elongatus; Dichelyne (D.) micropogonii; Cucullanus cassinensis (segundo PEREIRA \& COSTA 1996). Medidas em micrometros. Cucullanus fabregasi (segundo ForTES et al. 1993). Medidas em milímetros.

\begin{tabular}{|c|c|c|c|c|c|c|c|c|c|c|}
\hline & \multicolumn{2}{|c|}{ D. (C.) amaruincai } & \multicolumn{2}{|c|}{ D. (C.) elongatus } & \multicolumn{2}{|c|}{ D. (D.) micropogonii } & \multicolumn{2}{|c|}{ C. cassinensis } & \multicolumn{2}{|c|}{ C. fabregasi } \\
\hline & Macho & Fêmea & Macho & Fêmea & Macho & Fêmea & Macho & Fêmea & Macho & Fêmea \\
\hline Comprimento & $187,00-588,00$ & $141,00-772,00$ & $175,00-848,00$ & $124,00-657,00$ & $115,00-438,00$ & $155,00-835,00$ & $746,00-825,00$ & $258,00-1167,00$ & 9,22 & 10,24 \\
\hline Largura & $10,00-18,00$ & $7,00-23,00$ & $9,00-36,00$ & $6,00-38,00$ & $7,00-26,00$ & $9,00-37,00$ & $17,00-19,00$ & $18,00-30,00$ & 0,43 & 0,51 \\
\hline Anel nervoso & $13,00-27,00$ & $13,00-29,00$ & $18,00-34,00$ & $10,00-35,00$ & $8,00-32,00$ & $16,00-31,00$ & $32,00-34,00$ & $28,00-40,00$ & 0,40 & 0,40 \\
\hline Poro excretor & $56,00-59,00$ & $27,00-42,00$ & $35,00-62,00$ & $22,00-71,00$ & - & $33,00-51,00$ & - & - & - & - \\
\hline $\begin{array}{l}\text { Ventosa extremidade } \\
\text { posterior }\end{array}$ & $36,00-80,00$ & - & $25,00-90,00$ & - & - & - & $60,00-71,00$ & - & 0,11 & - \\
\hline $\begin{array}{l}\text { Comprimento da } \\
\text { ventosa }\end{array}$ & $3,75-1,25$ & - & $36,00-160,00$ & - & - & - & - & - & 0,25 & - \\
\hline Espiculo direito & $24,00-96,00$ & - & $22,00-69,00$ & - & $16,00-44,00$ & - & $52,00-68,00$ & - & 0,23 & - \\
\hline Espiculo esquerdo & $21,00-104,00$ & - & $20,00-69,00$ & - & $15,00-45,00$ & - & $57,00-72,00$ & - & 0,23 & - \\
\hline Cloaca & $9,00-12,00$ & - & $7,00-15,00$ & - & $6,00-12,00$ & - & $11,00-13,00$ & - & 0,11 & - \\
\hline Gubernáculo & $3,00-5,00$ & - & $1,80-5,00$ & - & $18,00-40,00$ & - & $4,00-5,00$ & - & 0,10 & - \\
\hline Esōfago & $44,00-68,00$ & $36,00-70,00$ & $46,00-93,00$ & $28,00-91,00$ & $29,00-84,00$ & $37,00-72,00$ & $86,00-88,00$ & $69,00-112,00$ & - & - \\
\hline Ceco intestinal & $15,00-33,00$ & $12,00-27,00$ & $13,00-40,00$ & $8,00-39,00$ & $9,00-28,00$ & $8,00-36,00$ & - & - & - & - \\
\hline Vulva & - & $56,00-264,00$ & - & $38,00-274,00$ & - & $72,00-190,00$ & - & 372,00 & - & 5,95 \\
\hline Ovos & - & $0,75 \times 1,00$ & - & $1,25 \times 0,02$ & - & $1,00 \times 1,25$ & - & $3,00 \times 5,00$ & - & $\begin{array}{c}0,05-0,09 \\
x\end{array}$ \\
\hline & & $2,75 \times 4,25$ & & $4,75 \times 7,50$ & & $3,25 \times 5,50$ & & $4,25 \times 5,50$ & & $0,03-0,05$ \\
\hline Reto & - & $6,00-16,00$ & - & $5,00-16,00$ & - & $5,00-15,00$ & - & 13,25 & - & 0,12 \\
\hline Ȧnus & - & $5,00-20,00$ & - & $7,00-22,0$ & - & $9,00-18,00$ & - & - & - & 0,22 \\
\hline
\end{tabular}




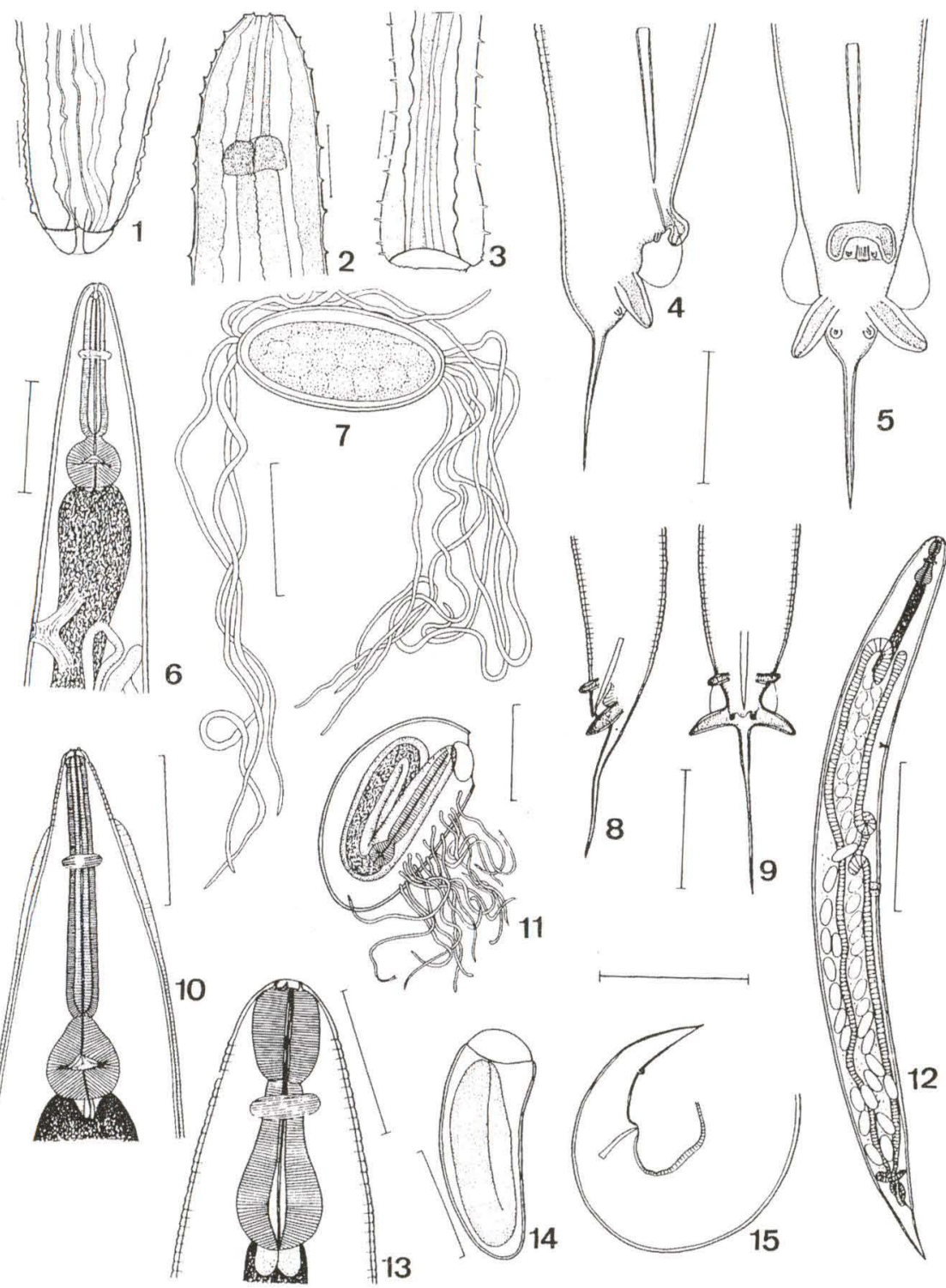

Figs 1-15. Eustrongylides ignotus: (1) cauda da fêmea (larva de quarto estágio); (2) extremidade anterior da larva; (3) extremidade posterior da cauda de macho imaturo (segundo REGO \& VICENTE 1988). Ichthyouris brasiliensis: (4) cauda do macho, vista lateral; (5) cauda do macho, vista ventral; (6) extremidade anterior; (7) ovo (segundo MORAVEC et al. 1992a). Ichthyouris laterifilamenta: (8) extremidade caudal do macho, vista lateral; (9) extremidade posterior do macho, vista ventral; (10) extremidade anterior; (11) ovo (segundo MoRAvecet al. 1992b). Travnema araujoi: (12) fêmea total; (13) extremidade anterior. (14) ovo; (15) extremidade caudal do macho, vista lateral (segundo Moravecet al. 1992b). Valores das escalas (mm): Figs 4, 5, 7, 8, 9, $11=0,05$; Figs 2, $14=0$, 1; Figs $1,3,6,10,13,15=0,2$; Fig. $12=1,0$. 


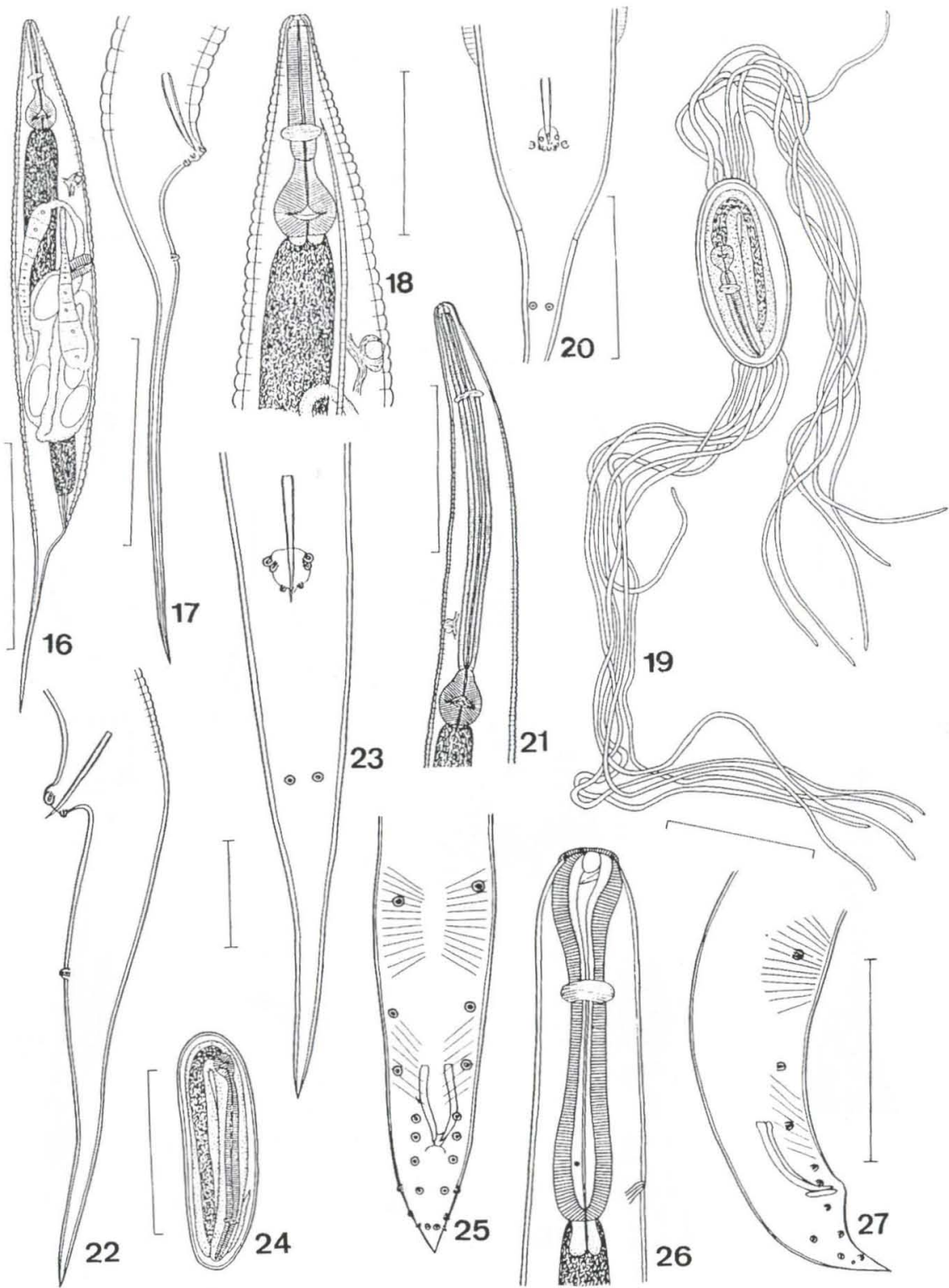

Figs 16-27. Brasilnema pimelodellae: (16) fêmea total; (17) cauda do macho, vista lateral. (18) extremidade anterior; (19) ovo; (20) extremidade caudal do macho, vista ventral (segundo MORAVEC et al. 1992a). Parasynodontisia petterae: (21) extremidade anterior; (22) extremidade caudal do macho, vista lateral; (23) extremidade caudal do macho, vista ventral; (24) ovo (segundo MORAVEC et al. 1992a). Cucullanus brevispiculus: (25) extremidade caudal do macho, vista ventral; (26) extremidade anterior; (27) extremidade caudal do macho, vista lateral (segundo MORAVEC et al. 1993a). Valores das escalas (mm): Figs 20-23 $=0,05$; Figs $17,19=0,1$; Figs $18,24=0,2$; Figs $21,25-27=$ 0,4 ; Fig. $16=0,5$. 

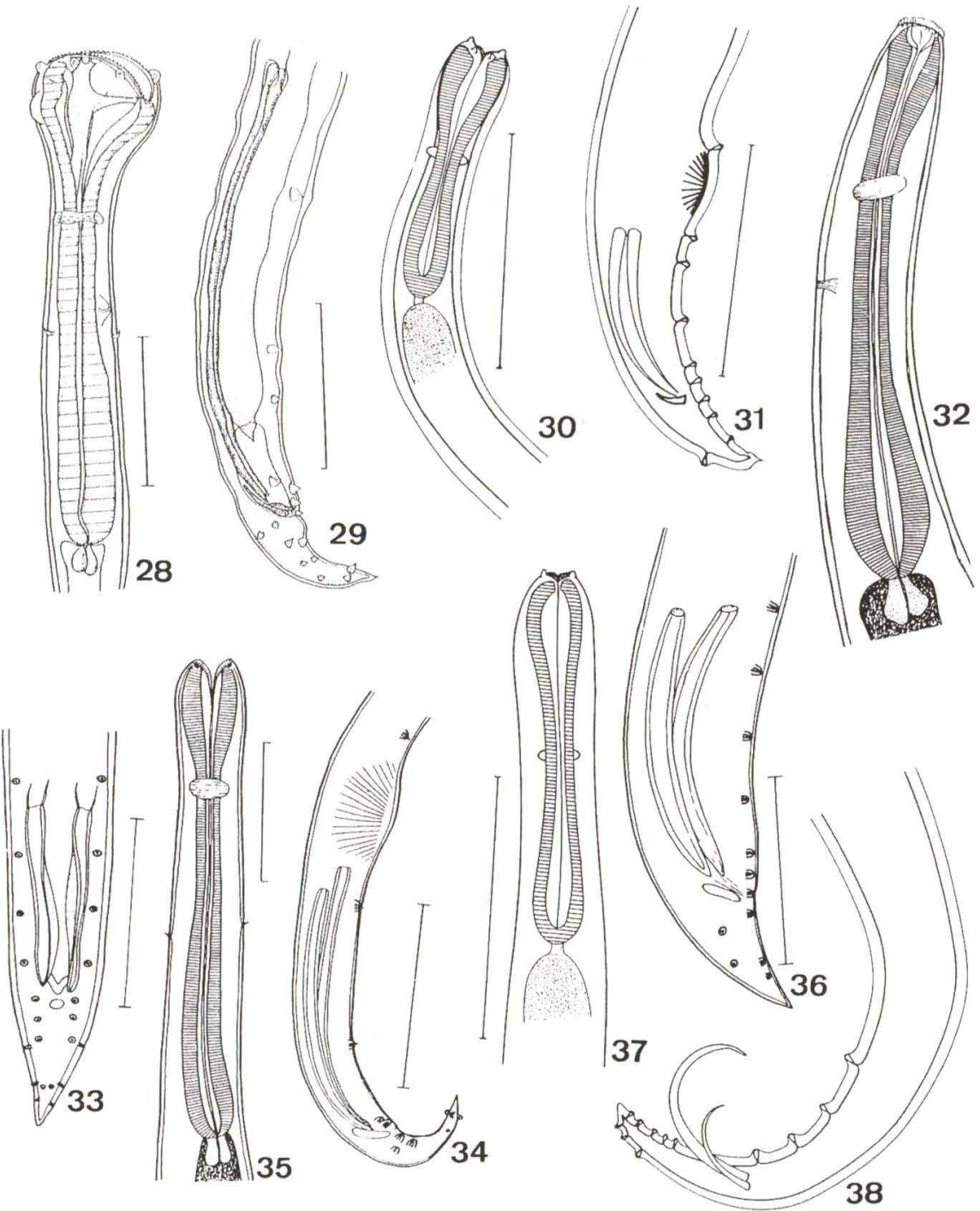

Figs 28-38. Cucullanus grandistomis: (28) extremidade anterior; (29) extremidade caudal do macho, vista lateral (segundo FERRAZ \& THATCHER 1988). Cucullanus patoi: (30) extremidade anterior; (31) extremidade caudal do macho, vista lateral (segundo ForTeset al. 1992). Cucullanus pimelodellae: (32) extremidade anterior; (33) extremidade caudal do macho, vista ventral; (34) extremidade caudal do macho, vista lateral (segundo MORAVEC et al. 1993a). Cucullanus riograndensis (35) extremidade anterior; (36) extremidade caudal do macho (segundo FORTES et al. 1993). Cucullanus pseudoplatystomae (37) extremidade anterior; (38) extremidade caudal do macho, (segundo MORAVEC et al. 1993a). Valores das escalas (mm): Figs $28,29,33,36=0,2$; Figs $32,35=0,3$; Fig. $34=0,4$; Figs $30,31,37,38=0,5$. 


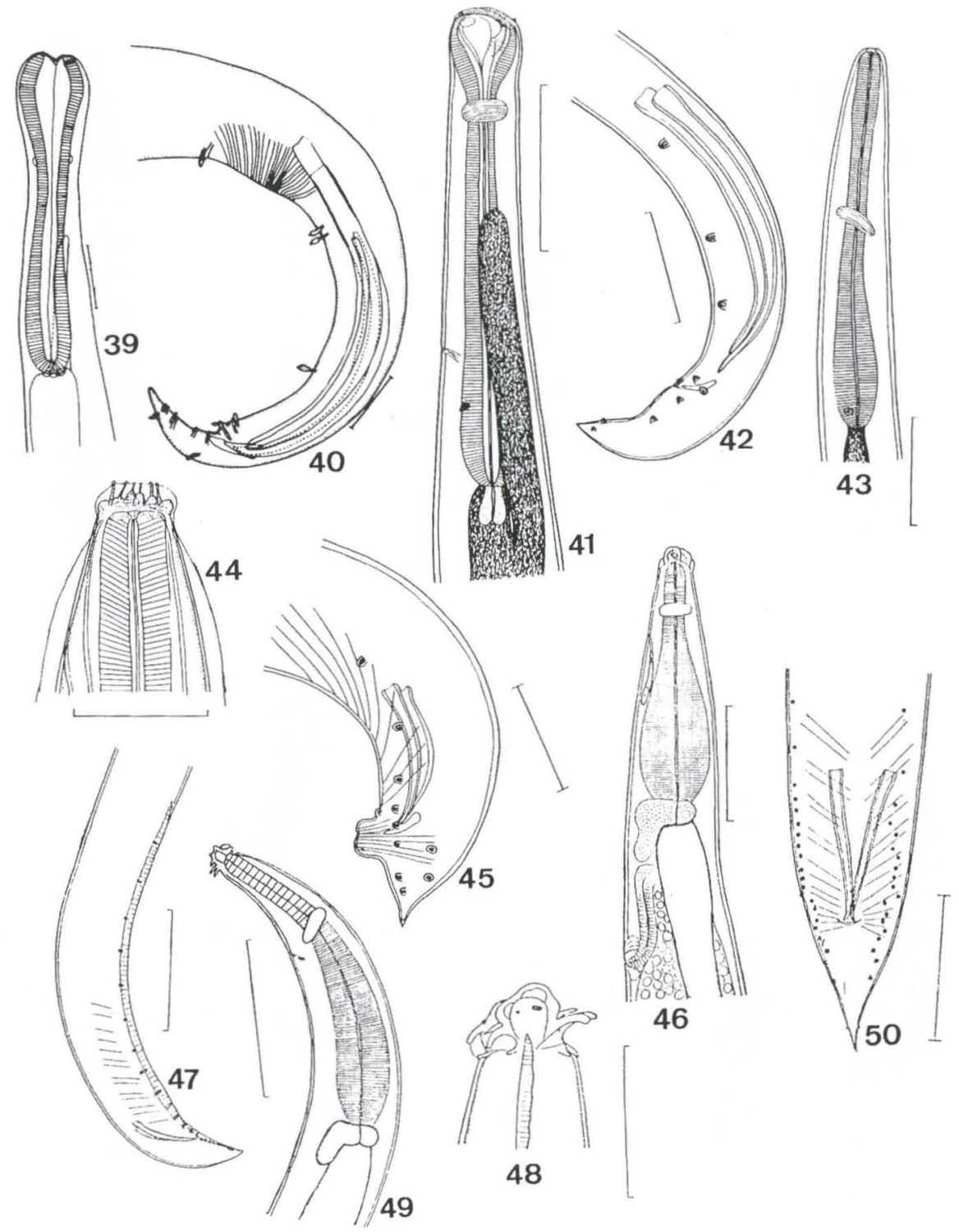

Figs 39-50. Dichelyne (C.) elongatus: (39) extremidade anterior; (40) extremidade caudal do macho (segundo PINTo et al. 1992). Dichelyne leporini: (41) extremidade anterior; (42) extremidade caudal do macho (segundo MORAVEC et al. 1993a). Neoparaseuratum travassosi: (43) extremidade anterior; (44) extremidade cefálica; (45) extremidade caudal do macho, (segundo MoRAVEc et al. 1993a). Raphidascaris (S.) hypostomi: (46) extremidade anterior; (47) extremidade caudal do macho, (segundo MoRAvec et al. 1993a). Raphidascaris (S.) mahnerti: (48) extremidade cefálica; (49) extremidade anterior do macho; (50) extremidade caudal do macho, (segundo MORAVEC et al. 1990). Valores das escalas (mm): Fig. $44=0,05$; Figs $40,43,48=0,1$; Fig. $39=0,15$; Figs $42,47,45,50=$ 0,2 ; Fig. $49=0,4$; Figs $41,46=0,5$. 

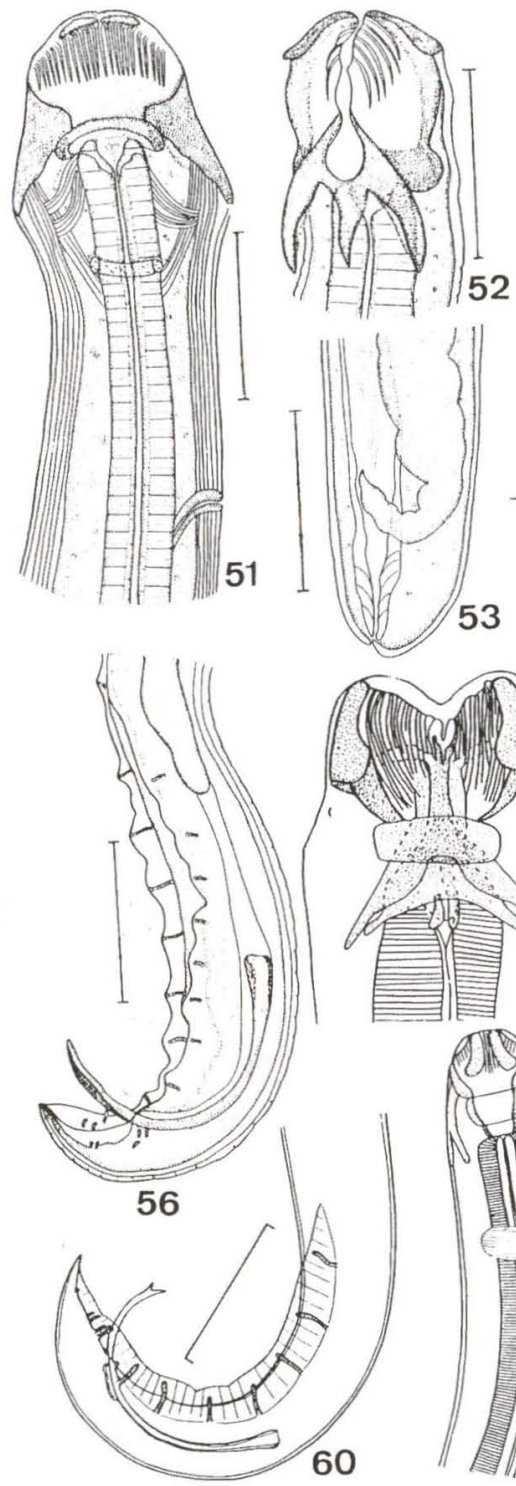

53
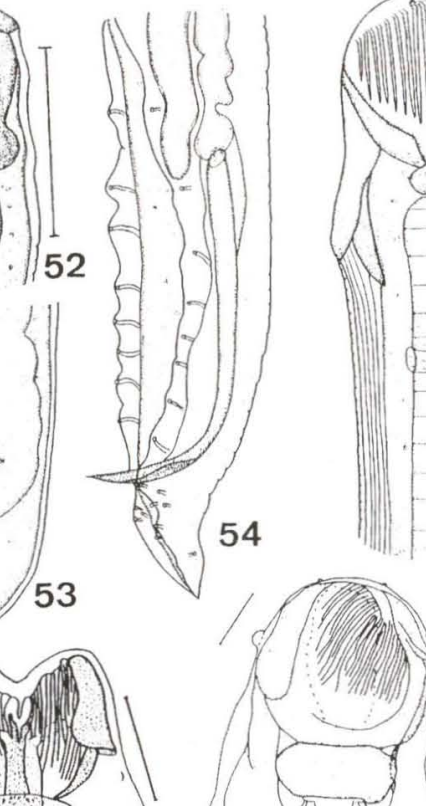

,
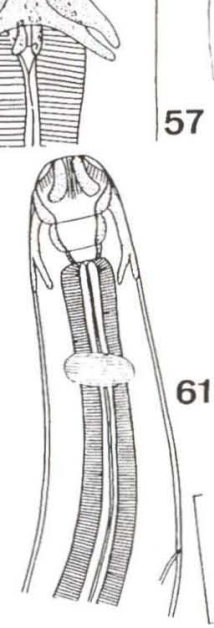

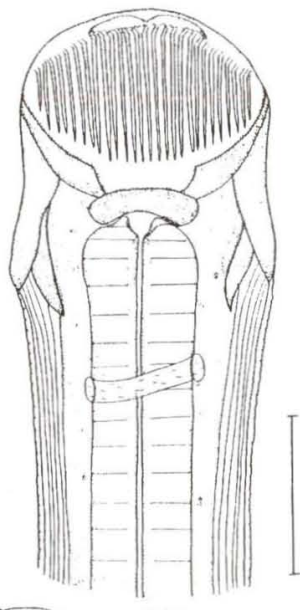

55

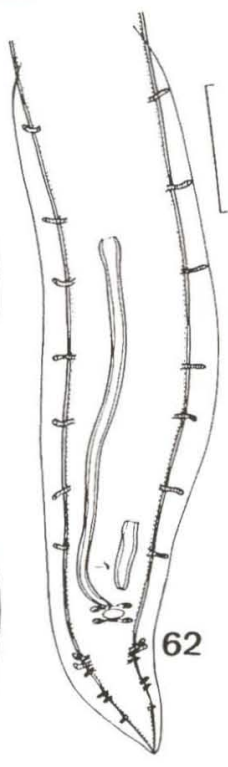

Figs 51-62. Camallanus acaudatus: (51) extremidade anterior; (52) extremidade cefálica; (53) extremidade caudal da fêmea; (54) extremidade caudal do macho, (segundo FERRAZ\& THATCHER1990). Camallanus tridentatus: (55) extremidade anterior; (56) extremidade caudal do macho, (segundo FERRAZ\& THATCHER 1990). Oncophora melanocephala: (57) extremidade cefálica, vista ventral; (58) extremidade cefálica, vista latero-ventral; (59) extremidade caudal do macho (segundo PINTO et al. 1988). Paracamallanus amazonensis: (60) extremidade caudal do macho; (61) extremidade anterior; (62) extremidade caudal do macho, (segundo Moravecet al. 1993a). Valores das escalas (mm): Figs $58,62=0,05$; Figs $51,52,54-57,60,61=0,1$; Fig. $59=0,2$; Fig. $53=0,5$. 


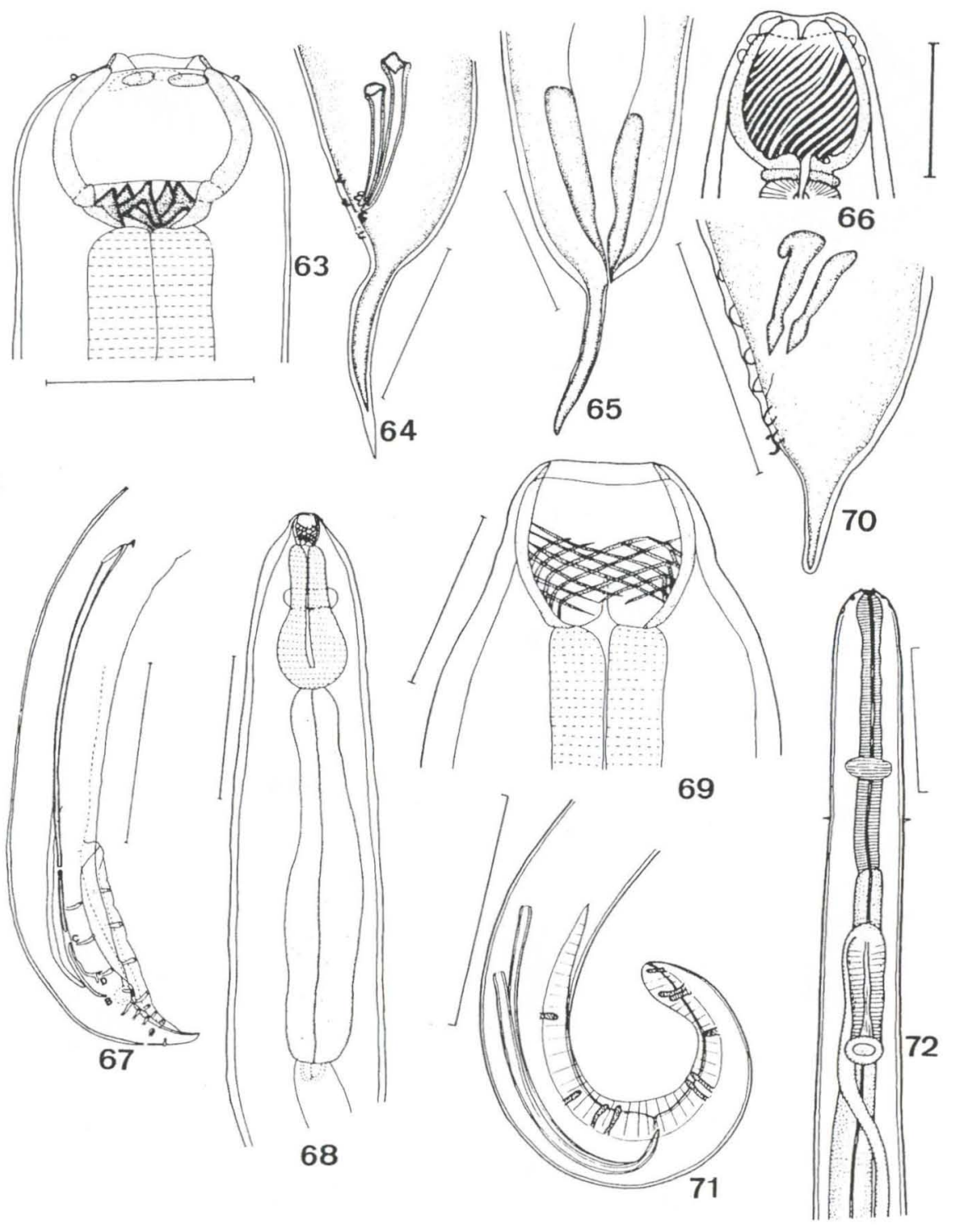

Figs 63-72. Procamallanus (P.) annipetterae: (63) cápsula bucal; (64) extremidade caudal do macho; (65) extremidade caudal da fêmea (segundo KOHN \& FERNANDES 1988a). Procamallanus (S.) freitasi: (66) cápsula bucal; (67) extremidade caudal do macho (segundo MOREIRA et al. 1991). Procamallanus (S.) pintoi: (68) extremidade anterior; (69) cápsula bucal; (70) extremidade caudal do macho (segundo KOHN \& FERNANDES 1988a). Guyanema raphidioni: (71) extremidade caudal do macho; (72) extremidade anterior (segundo Moravec et al. 1993c). Valores das escalas (mm): Figs 66, $69=0,05$; Figs $71,72=0,1$; Figs $63-65,67,68,70=0,2$. 

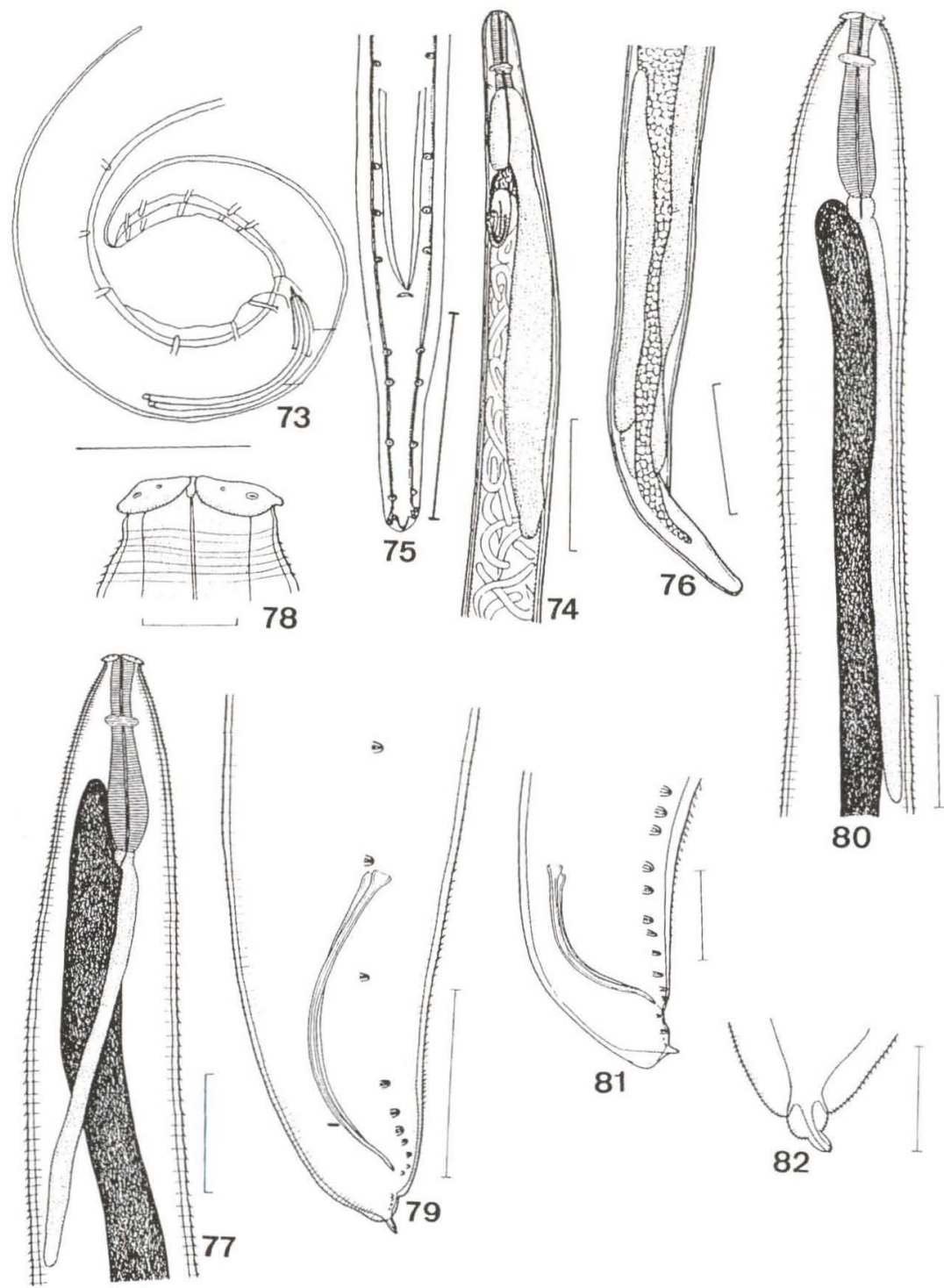

Figs 73-82. Travassosnema travassosi: (73) extremidade caudal do macho (segundo Costaet al. 1991). Travassosnema travassosi paranaensis: (74) extremidade anterior; (75) extremidade caudal do macho; (76) extremidade caudal da fêmea (segundo MorAvecet al. 1993c). Goezia brasiliensis: (77) extremidade anterior do macho; (78) extremidade cefálica, vista lateral; (79) extremidade caudal do macho, vista lateral. Goezia brevicaeca: (80) extremidade anterior do macho; (81) extremidade posterior do macho; (82) extremidade caudal da fêmea, vista ventral (segundo MoRAVEC et al. 1994). Valores das escalas (mm): Fig. $73=0,05$; Figs 76, 78, $82=0,1$; Figs $74,81=0,2$; Fig. $75=0,3$; Fig. $79=0,4 ;$ Figs $77,80=0,5$. 

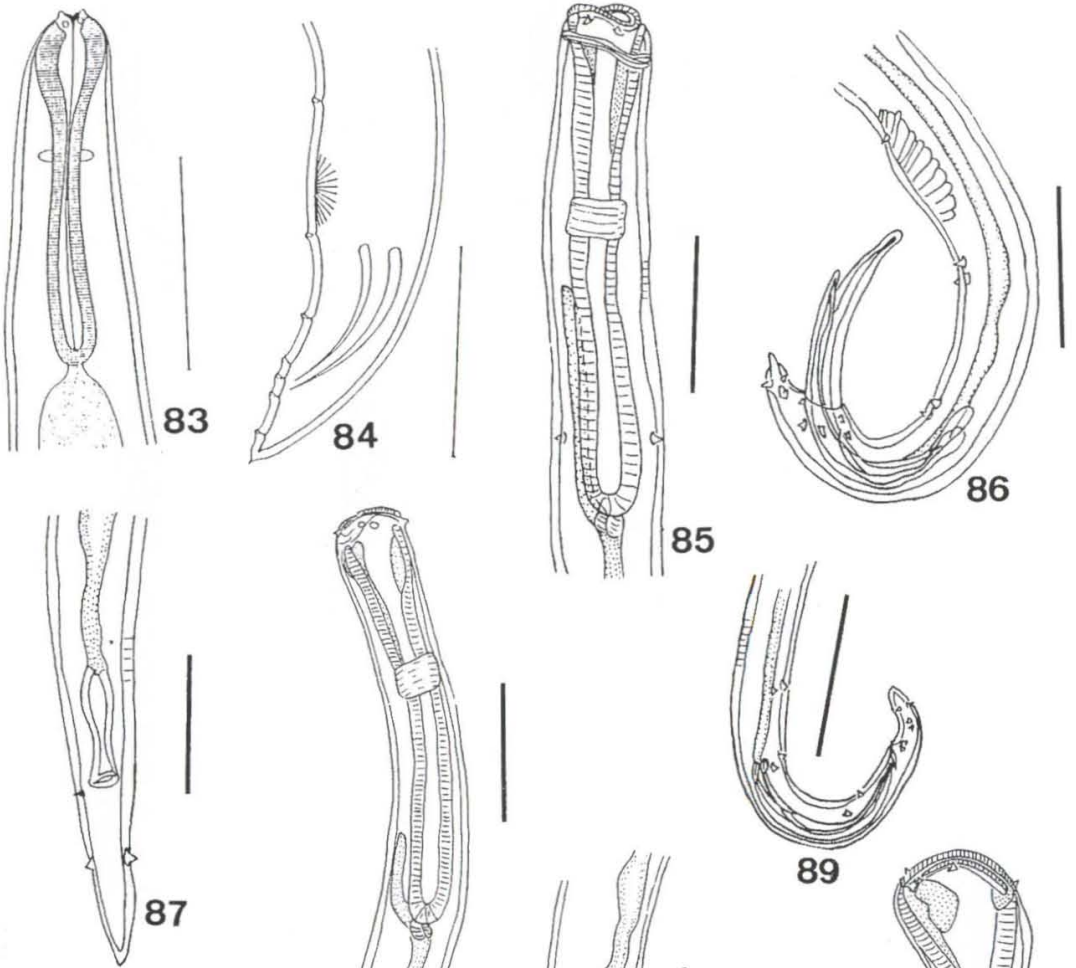

85
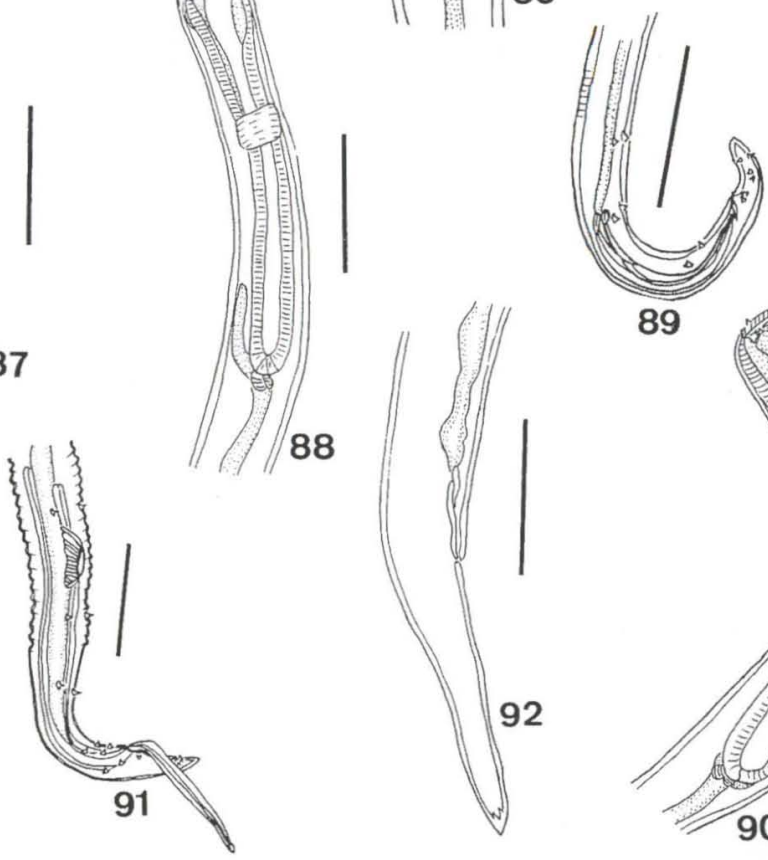

88
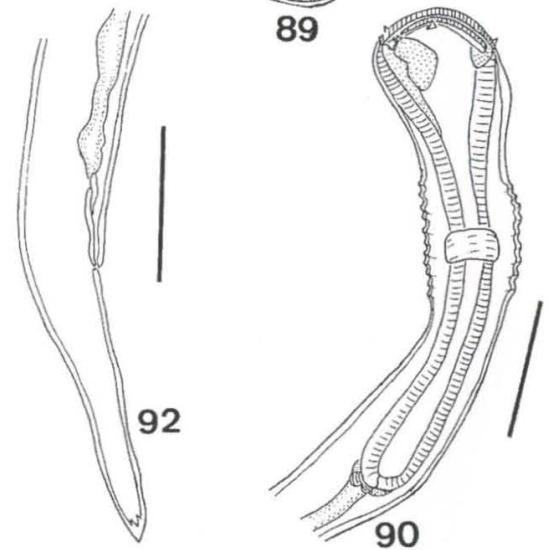

Figs 83-92. Cucullanus fabregasi: (83) extremidade anterior, vista ventral; (84) extremidade posterior do macho, vista lateral (segundo FORTES et al. 1993). Dichelyne (C.) amaruincai: (85) região anterior; (86) regiāo posterior do macho; (87) região posterior da fêmea. Dichelyne (D.) micropogonii: (88) região anterior; (89) região posterior do macho. Cucullanus cassinensis: (90) região anterior; (91) região posterior do macho; (92) regiāo posterior da fêmea (segundo PEREIRA \& CoSTA 1996). Valores das escalas (mm): Fig. $87=0,09$; Figs $88,91=0,15$; Figs $85,86,89,92=0,20$; Fig. $90=0,25$; Figs $83,84=0,5$. 


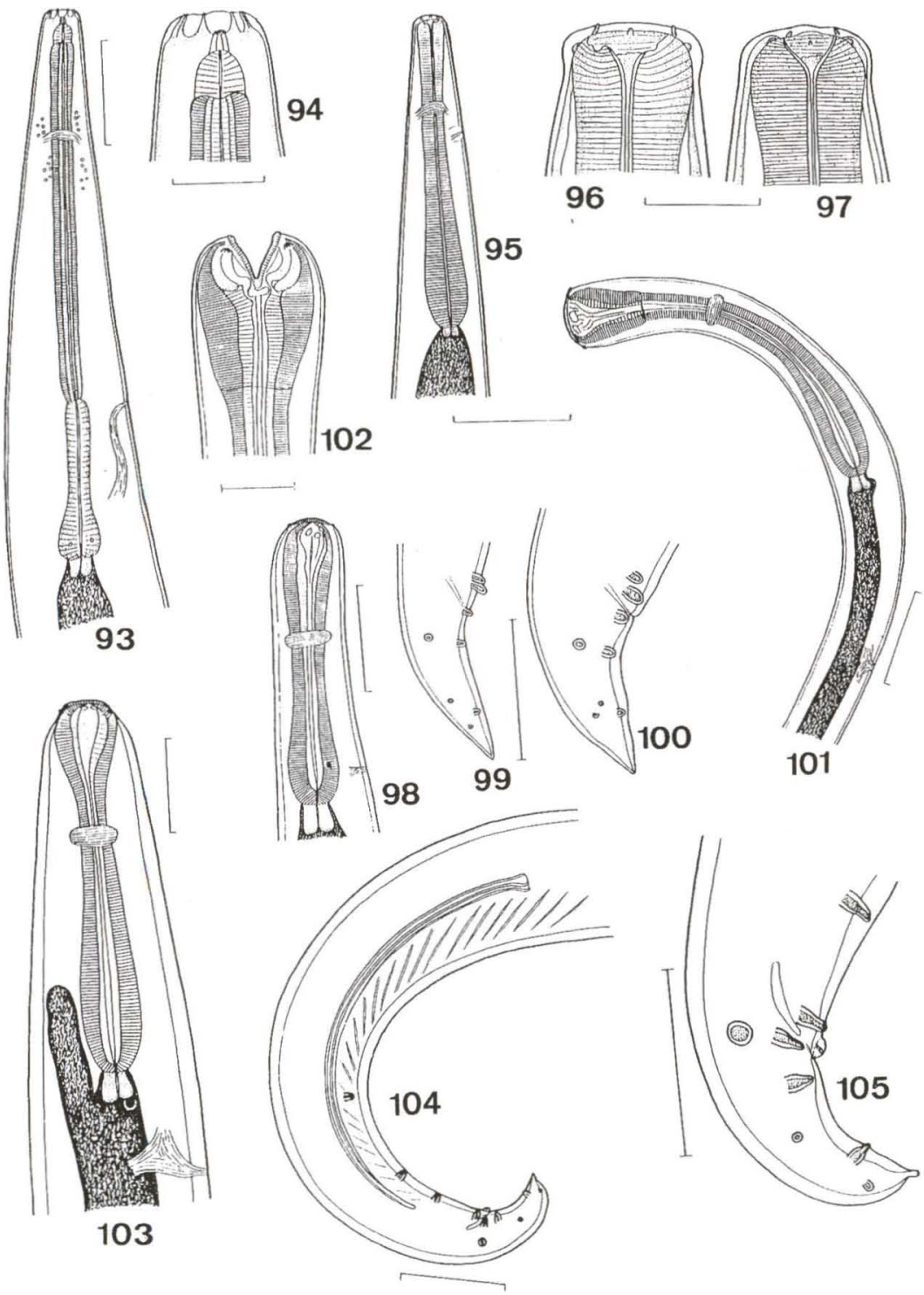

Figs 93-105. Spectatus sp.: (93) fêmea jovem; (94) extremidade anterior, vista lateral. Seuratoidea sp.: (95) extremidade anterior; (96) extremidade cefálica, vista dorsal; (97) extremidade cefálica, vista lateral. Cucullanus pinnai pterodorasi: (98) extremidade anterior, vista lateral; (99) cauda do macho menor; (100) cauda do macho maior. Cucullanus rhamphichthydis: (101) extremidade anterior; (102) extremidade cefálica. Dichelyne pimelodi. (103) extremidade anterior; (104) extremidade posterior; (105) cauda do macho, vista lateral (segundo MORAVEC et al. 1997a). Valores das escalas (mm): Figs 93, 98, 104 = 0,3; Figs 96, 97 = 0,05; Fig. 94 = 0,1; Figs 95, 99-103, 105. 


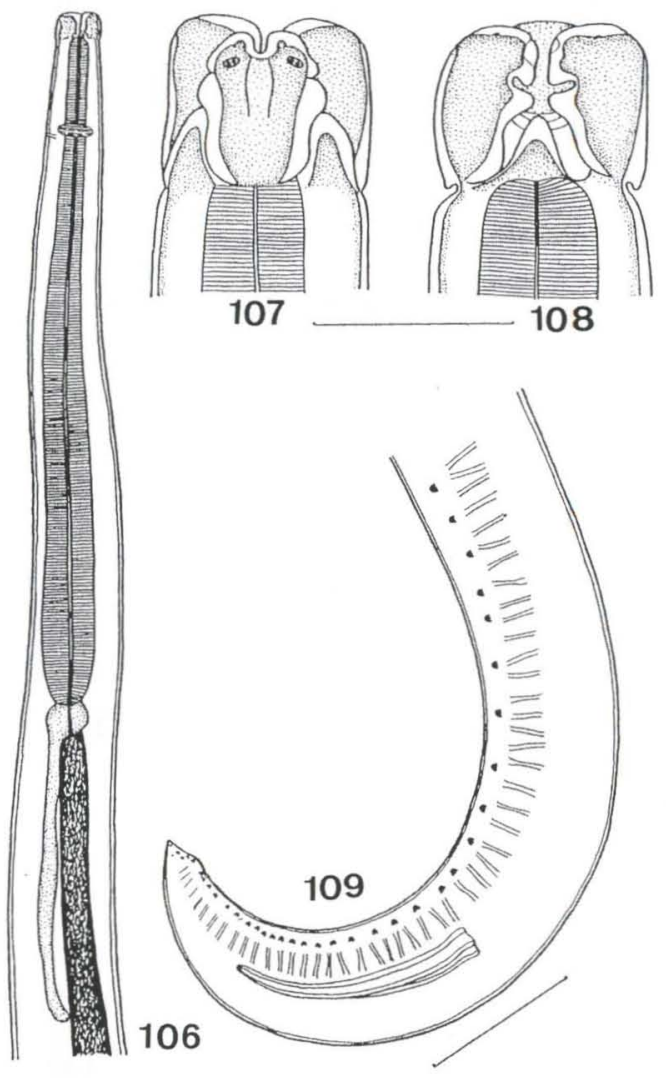

Figs 106-109. Raphidascaroides brasiliensis: (106) extremidade anterior, (107) extremidade cefálica, vista dorsal, (108) extremidade cefálica, vista ventral, (109) extremidade caudal do macho (Segundo Moravec\& Thatcher 1997b). Valores das escalas (mm): Fig. 106=0,5; Figs 107, $108=0,2 ;$ Fig. $109=$ 1,0 .

Revta bras. Zool. 16 (3): $561-610,1999$ 


\section{ÍNDICE}

Acestrorhamphus macrolepis

573,580

Acestrorhynchus lacustris

$576,577,580$

Acuariidae 564,576

Acuariinae sp.

$564,576,585$

Acuarioidea 564,576

Ageneiosus valenciennesi

$573,578,580$

Amplicaecum sp. $563,574,581,582$

Ancistrus cirrhosus 571,580

Anisakidae $563,564,571$

Ascarididae 574

Ascaridoidea $563,564,571$

Astianax fasciatus fasciatus . 576,581

Astyanax bimaculatus lacustris 579,580

Astyanax bimaculatus schubarti. 579,581

Atractidae 562,578

Auchenipterus nuchalis 568,581

Auchenipterus nigripinnis 566,581

Bergiaria sp. 573,581

Bergiaria westmanni $.575,581$

Brasilnema pimelodellae

Brycon erythropterum

Brycon hilarii

$562,566,583,589,598$

Camallanidae. $566,572,573,581$

Camallanoidea. $563,565,574,579$

Camallanus acaudatus. $563,565,574,579$

Camallanus tridentatus $563,574,582,592,601$

Capillaria sp. $563,574,582,592,601$

Contracaecum sp. $562,565,583$

Corydoras paleatus. $563,573,580,581,583,584$

Cosmocercoidea 576,581

Cosmoxynema vianai $562,571,578$ $.579,584$

Cosmoxynemoides aguirrei

Crenicichla haroldoi

$562,579,581,584$

Crenicichla lepidota 580,581

Cucullanidae $573,580,581$

Cucullanus brevispiculus

$562,565,568,578$

Cucullanus cassinensis $562,568,581,590,598$

Cucullanus debacoi $562,568,582,596,604$

Cucullanus fabregasi. $562,568,583$

Cucullanus grandistomis $562,568,583,596,604$

Cucullanus patoi $562,569,584,590,599$

Cucullanus pimelodellae $562,569,583,590,599$

Cucullanus pinai pinai. $562,569,583,590$

Cucullanus pinai pterodorasi $562,578,582,583$

Cucullanus pinnai. $562,569,584,595,605$

Cucullanus pseudoplatystomae $562,580,583,584$

Cucullanus pulcherrimus $562,569,584,590,599$

Cucullanus rhamphichthydis $563,578,582$ $569,595,605$ 
Cucullanus riograndensis

$562,569,583,591,599$

Cucullanus sp.

$563,570,582,584$

Cucullanus zungaro

$563,578,584$

Curimatus gilberti

567,581

Dichelyne (C.) amaruincai

$563,570,582,596,604$

Dichelyne (C.) elongatus...

$563,570,582,591,596,600$

Dichelyne (C.) $\mathrm{sp}$

$563,571,582$

Dichelyne (D.) micropogonii.

$563,571,582,596,604$

Dichelyne leporini

$563,570,585,591,600$

Dichelyne pimelodi

$563,570,583,595,605$

Dioctophymatidae 562,566

Dioctophymatoidea 562,566

Dracunculoidea $564,565,577$

Eustrongylides ignotus

Galeocharax humeralis $66,581,583,584,585,597$

Galeocharax knerii $66,581,583,584,585,597$

Geophagus brasiliensis

Goezia brasiliensis

Goezia brevicaeca $573,581572,573,581,594$ $563,572,581,584,603$ $563,573,581,594,603$

Goezia sp. $563,573,581,594,603$

Guyanema raphidioni. $563,573,580,584,585$

Guyanema sp. $564,577,584,593,602$

Guyanemidae..... $564,577,584$

Hoplias malabaricus $564,565,577$

Hypophthalmus edentatus $566,573,574,581$

Hysterothylacium sp. $573,575,581$

Ichthyouris brasiliensis $582,583,584,585$
$567,584,589,597$

Ichthyouris laterifilamenta

Klossinemella iheringi....

$562,567,585,589,597$
578,582

Leporinus copelandii ... 574,582

Leporimus fasciatus. 580,582

Leporinus friderici..... 580,582

Leporinus piau. 580,582

Leporinus reinhardti. 580,582

Leporinus taeniatus 582

Loricaria laticeps 572,582

Loricaria sp. 578

Loricariichthys sp. $572,573,582$

Micropogonias furnieri $568,570,571,578,582$

Mylesinus paraschomburgkii 578,582

Mylopus asterias. 580,582

Neoparaseuratum travassosi $563,568,584,585,591,600$

Oncophora melanocephala $564,574,575,584,592,601$

Osteoglossum bicirrhosum 574,582

Oxyuridae .579

Oxyuroidea $562,564,566,579$

Pagrus pagrus $572,573,574,582$

Paracamallanus amazonensis

Paracamallamus sp.

Paralonchurus brasiliensis

$564,575,581$ $570,571,578,582$

Revta bras. Zool. 16 (3): 561 - 610, 1999 
Parasynodontisia petterae $562,566,567,584,589,598$

Paratrigon sp. 566,582

Paulicea luetkeni. $568,570,578,582$

Pharyngodonidae $562,564,566,579$

Piaractus mesopotamicus (= Mylossoma orbignyanus). 571,583

Pimelodella gracilis 578,583

Pimelodella lateristriga $566,569,583$

Pimelodus maculatus. 580,583

Pimelodus ornatus 578,583

Pimelodus ortmanni. $573,580,583$

Pimelodus sp. .583

Pinirampus pinirampu 566,583

Plagioscium squamosissimus 573,583

Plecostomus albopunctatus $.571,572,575,583$

Plecostomus commersoni. 571,583

Plecostomus derbyi $.565,571,572,583$

Potamotrigon motoro. 580,583

Priacanthus arenatus. 575,584

Procamallanus (P.) peraccuratus

Procamallanus (S.) annipetterae. $. .564,580,581,583$

Procamallanus (S.) freitasi... $564,575,583,593,602$

Procamallanus (S.) hilarii $.564,575,581,583,593,602$

Procamallanus (S.) iheringi. 564,581

Procamallanus (S.) inopinatus $564,579,585$

Procamallanus (S.) pimelodus

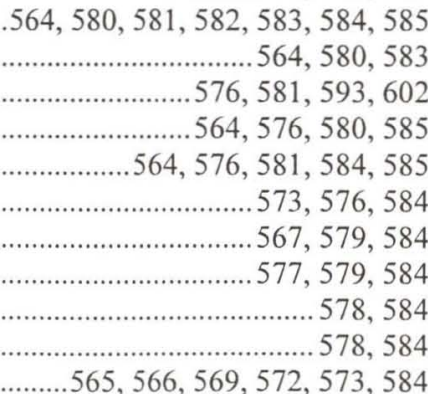

Procamallanus (S.) pintoi

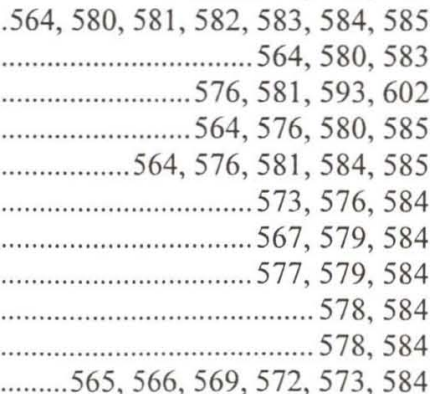

Procamallanus (S.) saofranciscencis

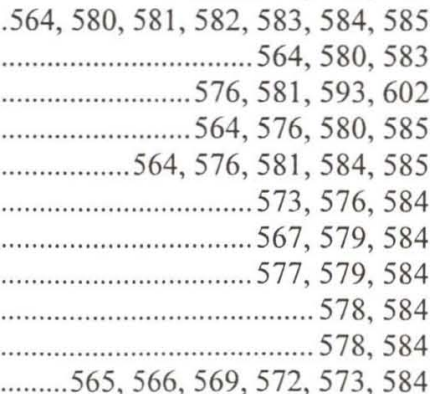

Procamallanus sp.

Prochilodus scrofa....

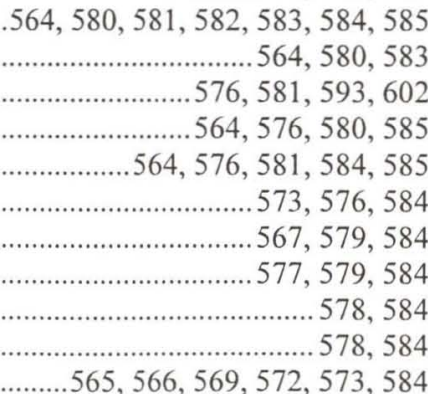

Pseudocurimata elegans

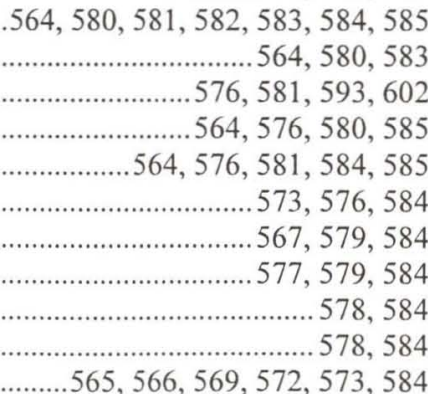

Pseudocurimata gilberti gilberti.

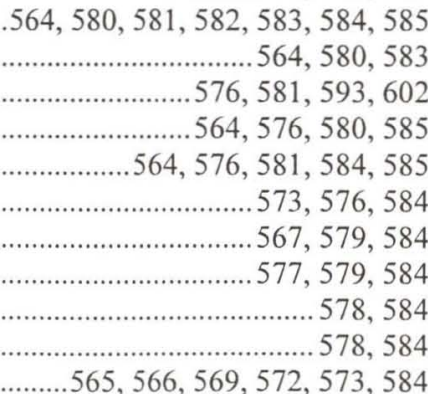

Pseudopimelodus roosevelti

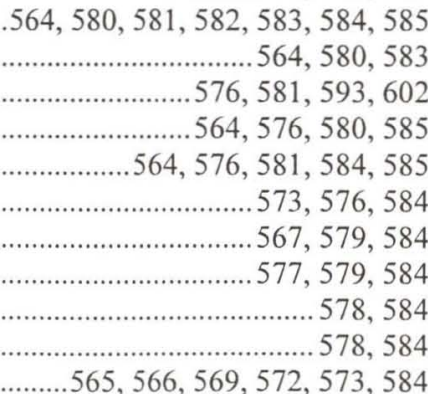

Pseudopimelodus zungaro

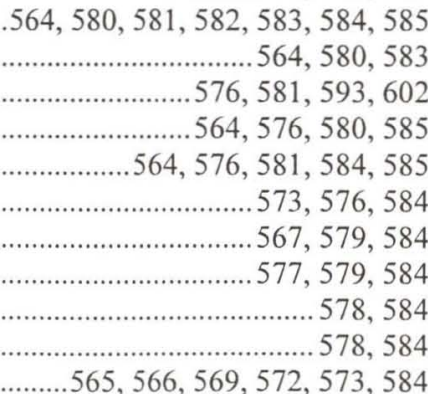

Pseudoplatistoma coruscans

Pseudoplatystoma fasciatus.

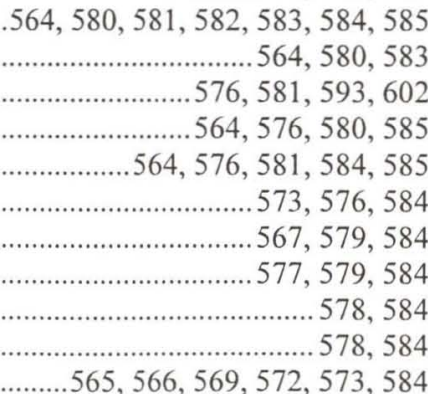

Pseudoras niger.

Pterodoras granulosus. 566,584 569,584

Pterygoplichthys aculeatus.... $568,569,570,572,573,575,580,584$

Quimperiidae. 567,584

Raphidascaris (S.) hypostomi $563,565,567$

Raphidascaris (S.) mahnerti Raphidascaris $\mathrm{sp}$. $563,571,580,583,592,600$
$\ldots \ldots .563,572,582,592,600$

Raphidascaroides brasiliensis $572,582,583$

Raphiodon vulpinus $563,572,606$

Rhamphichthys rostratus $566,573,577,584$

Rhinelepis aspera. 569

Rondonia rondoni 567,584

Salminus hilarii $562,578,585$ 576,585

Salminus maxillosus.

Schizodon fasciatus. 


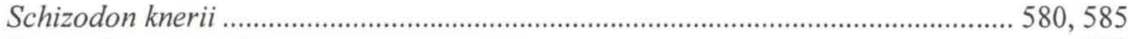

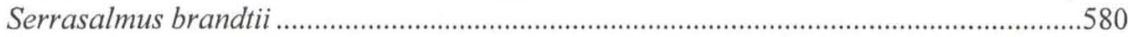

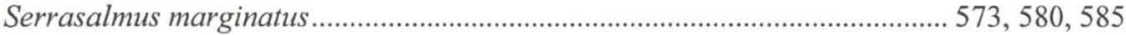

Serrasalmus nattereri ..................................................................................... 566, 585

Seuratoidea ….................................................... 563, 565, 567, 571, 578, 583, 595

Seuratoidea sp........................................................................................ 562, 568, 605

Spectatus sp.............................................................................. 571, 583, 595, 605

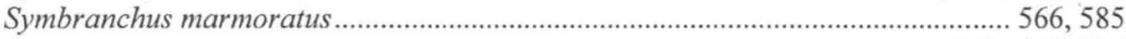

Terranova sp........................................................................................ 563, 574, 582

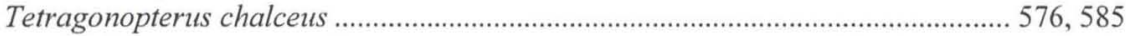

Trachydoras paraguayensis .................................................... 567, 568, 578, 580, 585

Travassosnema travassosi......................................................... 564, 577, 580, 594, 603

Travassosnema travassosi paranaensis ..................................... 564, 577, 580, 594, 603

Travnema araujoi …............................................................... 567, 581, 584, 589, 597

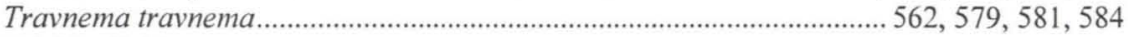

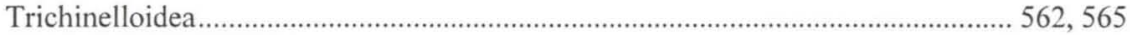

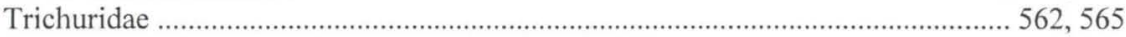

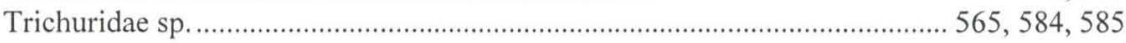

\section{NOTA}

Durante a fase final de editoração deste trabalho, obtivemos bibliografia pertinente ao assunto, publicada em 1998 (com distribuição em 1999) e que poderá, eventualmente, ser consultada como complementação aos dados apresentados nestecatálogo.

MORAVEC, F. 1998. Nematodes of freshwater fishes of the Neotropical Region. Praha, Academy of Sciences of the Czech Republic, 464p. 